Supporting Information for:

\title{
Synthesis of 1-Substituted Benzo[c]isoxazol-3(1H)-imines via Tandem Nitroso-Ene/Intramolecular Cyclizations of 2-Nitrosobenzonitrile
}

\author{
Jenna L. Jeffrey, Sean P. McClintock, and Michael M. Haley* \\ Department of Chemistry, University of Oregon, Eugene, Oregon 97403-1253 \\ haley@uoregon.edu
}

Table of Contents

Page

Experimental Procedures

$\mathrm{X}$-ray data for $\mathbf{9 a}$

S3

NMR spectra

S16

Computational Data

S27 


\section{Experimental Procedures}

2-Nitrosobenzonitrile (6): 2-Aminobenzonitrile (5 $\mathrm{g}, 42.3 \mathrm{mmol}$ ) was dissolved in $\mathrm{H}_{2} \mathrm{O} / \mathrm{CH}_{2} \mathrm{Cl}_{2}(4: 1 \mathrm{v} / \mathrm{v}, 0.1 \mathrm{M})$ and oxone $(52.1 \mathrm{~g}, 84.7 \mathrm{mmol})$ was added. ${ }^{10}$ The solution was stirred at $\mathrm{rt}$ until TLC indicated complete consumption of the starting material (ca. $3 \mathrm{~h}$ ). The aqueous layer was extracted with $\mathrm{CH}_{2} \mathrm{Cl}_{2}(3 \mathrm{X} 30 \mathrm{~mL})$ and washed successively with $10 \% \mathrm{HCl}$, $10 \% \mathrm{NaHCO}_{3}$ and brine. The combined organics were dried $\left(\mathrm{MgSO}_{4}\right)$, filtered, and concentrated. The crude product was purified over a pad of silica gel $\left(1: 1\right.$ hexanes $\left./ \mathrm{CH}_{2} \mathrm{Cl}_{2}\right)$ furnishing 6 (3.00 g, $65 \%)$ as a light yellow solid. ${ }^{1} \mathrm{H}$ NMR $\left(\mathrm{CDCl}_{3}\right) \delta 8.05(\mathrm{~d}, J=7.2 \mathrm{~Hz}, 1 \mathrm{H}), 7.86(\mathrm{t}, J=7.8 \mathrm{~Hz}$, $1 \mathrm{H}), 7.77(\mathrm{t}, J=7.8 \mathrm{~Hz}, 1 \mathrm{H}), 6.08(\mathrm{~d}, J=7.8 \mathrm{~Hz}, 1 \mathrm{H}) ;{ }^{13} \mathrm{C} \mathrm{NMR}\left(\mathrm{CDCl}_{3}\right) \delta$ 162.0, 135.6, 134.6, 133.7, 116.8, 114.0, 112.3; IR (NaCl) 2231, 1499, 1150, 1080, 813, $773 \mathrm{~cm}^{-1}$; HRMS (EI) for $\mathrm{C}_{7} \mathrm{H}_{4} \mathrm{~N}_{2} \mathrm{O}$ : calcd 132.0324, found 132.0326 .

1-(2,3-Dimethylbut-3-en-2-yl)benzo[c]isoxazol-3(1H)-one (8): 7 a $(65 \mathrm{mg}, 0.30 \mathrm{mmol})$ was dissolved in THF $(15 \mathrm{~mL}, 0.03 \mathrm{M})$ and $p-\mathrm{TsOH} \bullet \mathrm{H}_{2} \mathrm{O}(0.57 \mathrm{~g}, 3 \mathrm{mmol})$ was added. The solution was stirred at $\mathrm{rt}$ for $24 \mathrm{~h}$. The solvent was removed in vacuo and the crude mixture of 7a and 8 was purified by vacuum filtration through a pad of deactivated silica gel, eluting with 1:1 EtOAc/ $\mathrm{CH}_{2} \mathrm{Cl}_{2}$. Removal of the solvent in vacuo furnished a 1:1 mixture of $\mathbf{7 a}$ and $\mathbf{8}$, which were separated by column chromatography to afford $\mathbf{8}(32 \mathrm{mg}, 50 \%)$ as a yellow oil: ${ }^{1} \mathrm{H}-\mathrm{NMR}$ $\left(\mathrm{CDCl}_{3}\right) \delta 7.82(\mathrm{dd}, J=6.9,6.6 \mathrm{~Hz}, 1 \mathrm{H}), 7.53(\mathrm{dt}, J=8.4,1.2 \mathrm{~Hz}, 1 \mathrm{H}), 7.20(\mathrm{~m}, 2 \mathrm{H}), 5.13(\mathrm{~d}, J=$ $2.4 \mathrm{~Hz}, 2 \mathrm{H}), 2.04(\mathrm{~d}, J=0.6 \mathrm{~Hz}, 3 \mathrm{H}), 1.38(\mathrm{~s}, 6 \mathrm{H}) ;{ }^{13} \mathrm{C} \mathrm{NMR}\left(\mathrm{CDCl}_{3}\right) \delta$ 168.23, 148.38, 134.75, 125.92, 123.88, 114.14, 113.97, 68.46, 23.38, 19.51; IR (NaCl) 3501, 3094, 2987, 1755, 1643 , 1612, 1515, 1462, 1380, 1364, 1332, 1300, 1259, 1213, 1151, 1115, 1075, 1017, 972, 951, 911, 804, 787, 759, 729, 681, $627 \mathrm{~cm}^{-1}$; HRMS (EI) for $\mathrm{C}_{13} \mathrm{H}_{15} \mathrm{NO}_{2}$ : calcd 217.1103, found 217.1103.

(Z)-2-(3-Methylbut-3-en-2-ylideneamino)benzamide (10): $6(50 \mathrm{mg}, 0.378 \mathrm{mmol})$ was dissolved in freshly distilled THF $(15 \mathrm{~mL})$ and 2-methyl-2-butene $(1.89 \mathrm{~mL}, 3.78 \mathrm{mmol}, 2.0 \mathrm{M}$ in THF) was added. The solution was stirred at $\mathrm{rt}$ until TLC monitoring indicated complete consumption of the starting material. Crude $\mathbf{7 d}$ was converted to $\mathbf{1 0}$ by vacuum filtration through a pad of deactivated silica gel, eluting with EtOAc. Removal of the solvent in vacuo furnished $\mathbf{1 0}$ (59 mg, 77\%) as a light yellow solid: ${ }^{1} \mathrm{H}$ NMR $\left(\mathrm{CDCl}_{3}\right) \delta 8.21(\mathrm{dd}, J=8.1,1.5 \mathrm{~Hz}, 1 \mathrm{H}), 7.71(\mathrm{~s}$, $1 \mathrm{H}), 7.48(\mathrm{dt}, J=7.5,1.8 \mathrm{~Hz}, 1 \mathrm{H}), 7.26(\mathrm{dt}, J=7.8,1.2 \mathrm{~Hz}, 1 \mathrm{H}), 6.67(\mathrm{dd}, J=8.1,1.2 \mathrm{~Hz}, 1 \mathrm{H})$, $6.59(\mathrm{~s}, 1 \mathrm{H}), 5.82(\mathrm{~s}, 1 \mathrm{H}), 5.74(\mathrm{~s}, 1 \mathrm{H}), 2.14(\mathrm{~s}, 3 \mathrm{H}), 2.10(\mathrm{~s}, 3 \mathrm{H}) ;{ }^{13} \mathrm{C} \mathrm{NMR}\left(\mathrm{CDCl}_{3}\right) \delta 169.13$, 168.57, 149.19, 144.87, 132.12, 131.39, 124.51, 122.45, 120.19, 19.64, 16.97; IR (NaCl) 3344, 3181, 2977, 1666, 1613, 1447, 1370, 1151, 923, $787 \mathrm{~cm}^{-1}$; HRMS (EI) for $\mathrm{C}_{12} \mathrm{H}_{14} \mathrm{~N}_{2} \mathrm{O}$ : calcd 202.1106, found 202.1099. 
Molecular structure of 9a; ellipsoids drawn at the 30\% probability level.

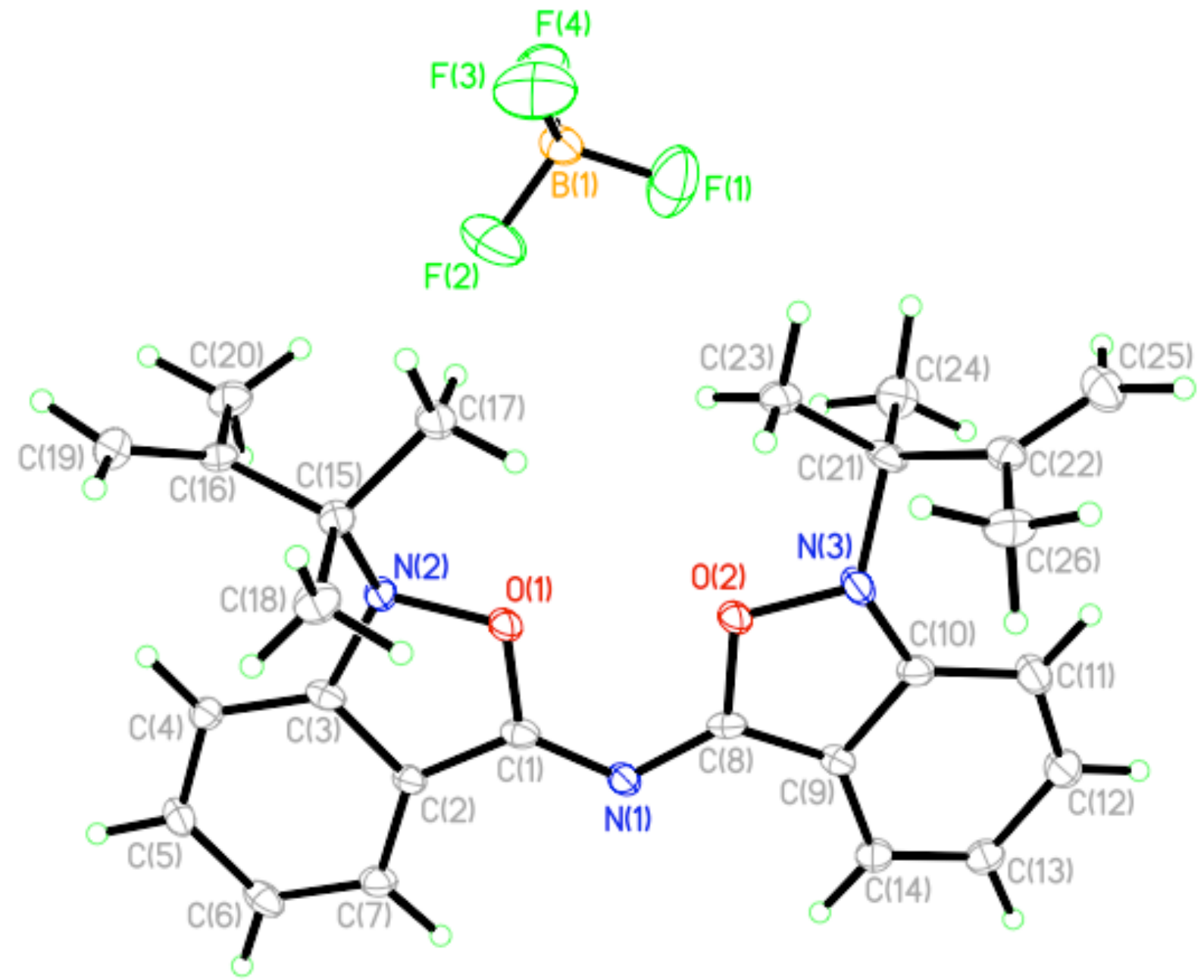


Table 1. Crystal data and structure refinement for $9 \mathbf{a}$.

Empirical formula

Formula weight

Temperature

Wavelength

Crystal system

Space group

Unit cell dimensions

Volume

Z

Density (calculated)

Absorption coefficient

$\mathrm{F}(000)$

Crystal size

Theta range for data collection

Index ranges

Reflections collected

Independent reflections

Completeness to theta $=25.00^{\circ}$

Absorption correction

Max. and min. transmission

Refinement method

Data / restraints / parameters

Goodness-of-fit on $\mathrm{F}^{2}$

Final R indices [I $>2 \operatorname{sigma(I)]~}$

$\mathrm{R}$ indices (all data)

Largest diff. peak and hole
C13 H15 B0.50 F2 N1.50 O

251.67

173(2) K

$0.71073 \AA$

Monoclinic

$\mathrm{P} 2(1) / \mathrm{n}$

$\mathrm{a}=10.710(2) \AA \quad \alpha=90^{\circ}$

$\mathrm{b}=10.795(2) \AA \quad \beta=93.700(4)^{\circ}$

$\mathrm{c}=21.519(5) \AA \quad \gamma=90^{\circ}$

2482.9(9) $\AA^{3}$

8

$1.347 \mathrm{Mg} / \mathrm{m}^{3}$

$0.105 \mathrm{~mm}^{-1}$

1056

$0.18 \times 0.12 \times 0.02 \mathrm{~mm}^{3}$

1.90 to $25.00^{\circ}$

$-12<=\mathrm{h}<=12,-12<=\mathrm{k}<=12,-25<=1<=25$

21628

$4357[\mathrm{R}(\mathrm{int})=0.1511]$

$99.9 \%$

Semi-empirical from equivalents

0.9979 and 0.9813

Full-matrix least-squares on $\mathrm{F}^{2}$

4357 / 0 / 445

0.993

$\mathrm{R} 1=0.0662, \mathrm{wR} 2=0.1235$

$\mathrm{R} 1=0.1478, w R 2=0.1608$

0.459 and -0.302 e. $\AA^{-3}$ 
Table 2. Atomic coordinates $\left(\mathrm{x} 10^{4}\right)$ and equivalent isotropic displacement parameters $\left(\AA^{2} \times 10^{3}\right)$ for 9 a. $U(e q)$ is defined as one third of the trace of the orthogonalized $U^{\mathrm{ij}}$ tensor.

\begin{tabular}{|c|c|c|c|c|}
\hline & $\mathrm{X}$ & $\mathrm{y}$ & $\mathrm{Z}$ & $\mathrm{U}(\mathrm{eq})$ \\
\hline $\mathrm{O}(1)$ & $8671(2)$ & $6591(3)$ & $4411(1)$ & $28(1)$ \\
\hline $\mathrm{O}(2)$ & $10022(2)$ & $8426(2)$ & $4098(1)$ & $27(1)$ \\
\hline $\mathrm{N}(1)$ & $10062(3)$ & $7624(3)$ & $5118(1)$ & $27(1)$ \\
\hline $\mathrm{N}(2)$ & $7962(3)$ & $5460(3)$ & $4411(1)$ & $26(1)$ \\
\hline $\mathrm{N}(3)$ & $10685(3)$ & $9349(3)$ & 3791(1) & $38(1)$ \\
\hline $\mathrm{C}(1)$ & $9228(3)$ & $6756(4)$ & $4986(2)$ & $26(1)$ \\
\hline $\mathrm{C}(2)$ & $8818(3)$ & $5826(4)$ & $5390(2)$ & $23(1)$ \\
\hline$C(3)$ & $8006(3)$ & $5075(4)$ & $5027(2)$ & $24(1)$ \\
\hline$C(4)$ & $7428(3)$ & $4048(4)$ & $5283(2)$ & $26(1)$ \\
\hline$C(5)$ & $7711(4)$ & $3821(4)$ & $5908(2)$ & $30(1)$ \\
\hline$C(6)$ & $8550(4)$ & $4563(4)$ & $6273(2)$ & $34(1)$ \\
\hline$C(7)$ & $9119(4)$ & $5560(4)$ & $6020(2)$ & $30(1)$ \\
\hline $\mathrm{C}(8)$ & 10494(3) & $8403(4)$ & $4699(2)$ & $26(1)$ \\
\hline$C(9)$ & $11479(3)$ & $9248(4)$ & $4777(2)$ & $24(1)$ \\
\hline$C(10)$ & $11595(3)$ & $9789(4)$ & $4192(2)$ & $28(1)$ \\
\hline $\mathrm{C}(11)$ & $12557(4)$ & $10645(4)$ & $4092(2)$ & $37(1)$ \\
\hline$C(12)$ & $13365(4)$ & $10902(5)$ & $4594(2)$ & $39(1)$ \\
\hline$C(13)$ & $13244(4)$ & $10365(4)$ & $5190(2)$ & $35(1)$ \\
\hline$C(14)$ & $12311(4)$ & $9551(4)$ & $5288(2)$ & $30(1)$ \\
\hline$C(15)$ & $6786(3)$ & $5576(4)$ & $3994(2)$ & $26(1)$ \\
\hline$C(16)$ & $6338(3)$ & $4249(4)$ & $3860(2)$ & $27(1)$ \\
\hline$C(17)$ & $7132(4)$ & $6195(5)$ & $3388(2)$ & $35(1)$ \\
\hline $\mathrm{C}(18)$ & $5849(4)$ & $6376(5)$ & $4310(2)$ & $36(1)$ \\
\hline$C(19)$ & $5156(4)$ & $3921(5)$ & $3881(2)$ & $36(1)$ \\
\hline$C(20)$ & $7302(4)$ & $3352(5)$ & $3653(2)$ & $35(1)$ \\
\hline$C(21)$ & 10541(3) & $9332(4)$ & $3093(2)$ & $30(1)$ \\
\hline$C(22)$ & $10462(4)$ & $10686(4)$ & $2897(2)$ & $29(1)$ \\
\hline
\end{tabular}




\begin{tabular}{lllll}
$\mathrm{C}(23)$ & $9317(4)$ & $8656(5)$ & $2897(2)$ & $38(1)$ \\
$\mathrm{C}(24)$ & $11645(4)$ & $8626(5)$ & $2845(2)$ & $39(1)$ \\
$\mathrm{C}(25)$ & $11175(4)$ & $11160(6)$ & $2478(2)$ & $42(1)$ \\
$\mathrm{C}(26)$ & $9481(5)$ & $11455(5)$ & $3179(2)$ & $41(1)$ \\
$\mathrm{B}(1)$ & $9682(4)$ & $4893(6)$ & $1967(2)$ & $39(1)$ \\
$\mathrm{F}(1)$ & $10484(3)$ & $5873(3)$ & $2018(2)$ & $96(1)$ \\
$\mathrm{F}(2)$ & $9538(3)$ & $4454(3)$ & $2556(1)$ & $95(1)$ \\
$\mathrm{F}(3)$ & $8561(3)$ & $5210(3)$ & $1705(1)$ & $91(1)$ \\
$\mathrm{F}(4)$ & $10198(2)$ & $3968(3)$ & $1609(1)$ & $65(1)$ \\
\hline
\end{tabular}


Table 3. Bond lengths $[\AA]$ and angles $\left[{ }^{\circ}\right]$ for $9 \mathbf{a}$.

\begin{tabular}{|c|c|}
\hline $\mathrm{O}(1)-\mathrm{C}(1)$ & $1.352(4)$ \\
\hline $\mathrm{O}(1)-\mathrm{N}(2)$ & $1.438(4)$ \\
\hline $\mathrm{O}(2)-\mathrm{C}(8)$ & $1.357(4)$ \\
\hline $\mathrm{O}(2)-\mathrm{N}(3)$ & $1.413(4)$ \\
\hline $\mathrm{N}(1)-\mathrm{C}(1)$ & $1.313(5)$ \\
\hline $\mathrm{N}(1)-\mathrm{C}(8)$ & $1.337(5)$ \\
\hline $\mathrm{N}(2)-\mathrm{C}(3)$ & $1.387(4)$ \\
\hline $\mathrm{N}(2)-\mathrm{C}(15)$ & $1.503(4)$ \\
\hline $\mathrm{N}(3)-\mathrm{C}(10)$ & $1.346(4)$ \\
\hline $\mathrm{N}(3)-\mathrm{C}(21)$ & $1.500(4)$ \\
\hline$C(1)-C(2)$ & $1.416(5)$ \\
\hline$C(2)-C(3)$ & $1.391(5)$ \\
\hline$C(2)-C(7)$ & $1.402(5)$ \\
\hline$C(3)-C(4)$ & $1.401(6)$ \\
\hline$C(4)-C(5)$ & $1.380(5)$ \\
\hline $\mathrm{C}(4)-\mathrm{H}(4)$ & $0.95(3)$ \\
\hline$C(5)-C(6)$ & $1.406(6)$ \\
\hline $\mathrm{C}(5)-\mathrm{H}(5)$ & $0.99(4)$ \\
\hline$C(6)-C(7)$ & $1.367(6)$ \\
\hline $\mathrm{C}(6)-\mathrm{H}(6)$ & $0.99(3)$ \\
\hline $\mathrm{C}(7)-\mathrm{H}(7)$ & $0.99(3)$ \\
\hline$C(8)-C(9)$ & $1.396(5)$ \\
\hline $\mathrm{C}(9)-\mathrm{C}(10)$ & $1.401(5)$ \\
\hline $\mathrm{C}(9)-\mathrm{C}(14)$ & $1.409(5)$ \\
\hline $\mathrm{C}(10)-\mathrm{C}(11)$ & $1.410(6)$ \\
\hline $\mathrm{C}(11)-\mathrm{C}(12)$ & $1.368(6)$ \\
\hline $\mathrm{C}(11)-\mathrm{H}(11)$ & $0.96(3)$ \\
\hline $\mathrm{C}(12)-\mathrm{C}(13)$ & $1.421(6)$ \\
\hline $\mathrm{C}(12)-\mathrm{H}(12)$ & $0.98(4)$ \\
\hline $\mathrm{C}(13)-\mathrm{C}(14)$ & $1.357(6)$ \\
\hline
\end{tabular}




\begin{tabular}{|c|c|}
\hline $\mathrm{C}(13)-\mathrm{H}(13)$ & $1.00(4)$ \\
\hline $\mathrm{C}(14)-\mathrm{H}(14)$ & $0.96(4)$ \\
\hline$C(15)-C(18)$ & $1.518(6)$ \\
\hline$C(15)-C(17)$ & $1.531(6)$ \\
\hline$C(15)-C(16)$ & $1.532(6)$ \\
\hline$C(16)-C(19)$ & $1.318(5)$ \\
\hline$C(16)-C(20)$ & $1.504(6)$ \\
\hline $\mathrm{C}(17)-\mathrm{H}(17 \mathrm{~A})$ & $1.05(4)$ \\
\hline $\mathrm{C}(17)-\mathrm{H}(17 \mathrm{~B})$ & $1.01(4)$ \\
\hline $\mathrm{C}(17)-\mathrm{H}(17 \mathrm{C})$ & $1.02(4)$ \\
\hline $\mathrm{C}(18)-\mathrm{H}(18 \mathrm{~A})$ & $0.98(4)$ \\
\hline $\mathrm{C}(18)-\mathrm{H}(18 \mathrm{~B})$ & $0.97(4)$ \\
\hline $\mathrm{C}(18)-\mathrm{H}(18 \mathrm{C})$ & $1.02(4)$ \\
\hline $\mathrm{C}(19)-\mathrm{H}(19 \mathrm{~A})$ & $0.95(4)$ \\
\hline $\mathrm{C}(19)-\mathrm{H}(19 \mathrm{~B})$ & $0.99(4)$ \\
\hline $\mathrm{C}(20)-\mathrm{H}(20 \mathrm{~A})$ & $1.08(6)$ \\
\hline $\mathrm{C}(20)-\mathrm{H}(20 \mathrm{~B})$ & $0.99(4)$ \\
\hline $\mathrm{C}(20)-\mathrm{H}(20 \mathrm{C})$ & $0.99(4)$ \\
\hline $\mathrm{C}(21)-\mathrm{C}(22)$ & $1.523(6)$ \\
\hline$C(21)-C(24)$ & $1.530(6)$ \\
\hline $\mathrm{C}(21)-\mathrm{C}(23)$ & $1.535(6)$ \\
\hline$C(22)-C(25)$ & $1.321(6)$ \\
\hline$C(22)-C(26)$ & $1.498(6)$ \\
\hline $\mathrm{C}(23)-\mathrm{H}(23 \mathrm{~A})$ & $0.97(5)$ \\
\hline $\mathrm{C}(23)-\mathrm{H}(23 \mathrm{~B})$ & $1.02(5)$ \\
\hline $\mathrm{C}(23)-\mathrm{H}(23 \mathrm{C})$ & $1.04(4)$ \\
\hline $\mathrm{C}(24)-\mathrm{H}(24 \mathrm{~A})$ & $1.04(5)$ \\
\hline $\mathrm{C}(24)-\mathrm{H}(24 \mathrm{~B})$ & $1.01(5)$ \\
\hline $\mathrm{C}(24)-\mathrm{H}(24 \mathrm{C})$ & $1.06(4)$ \\
\hline $\mathrm{C}(25)-\mathrm{H}(25 \mathrm{~A})$ & $1.02(5)$ \\
\hline $\mathrm{C}(25)-\mathrm{H}(25 \mathrm{~B})$ & $0.97(4)$ \\
\hline $\mathrm{C}(26)-\mathrm{H}(26 \mathrm{~A})$ & $0.93(5)$ \\
\hline
\end{tabular}




\begin{tabular}{|c|c|}
\hline $\mathrm{C}(26)-\mathrm{H}(26 \mathrm{~B})$ & $1.11(5)$ \\
\hline $\mathrm{C}(26)-\mathrm{H}(26 \mathrm{C})$ & $1.01(4)$ \\
\hline $\mathrm{B}(1)-\mathrm{F}(3)$ & $1.337(5)$ \\
\hline $\mathrm{B}(1)-\mathrm{F}(1)$ & $1.363(6)$ \\
\hline $\mathrm{B}(1)-\mathrm{F}(2)$ & $1.370(6)$ \\
\hline $\mathrm{B}(1)-\mathrm{F}(4)$ & $1.398(6)$ \\
\hline $\mathrm{C}(1)-\mathrm{O}(1)-\mathrm{N}(2)$ & $108.3(3)$ \\
\hline $\mathrm{C}(8)-\mathrm{O}(2)-\mathrm{N}(3)$ & $107.0(3)$ \\
\hline $\mathrm{C}(1)-\mathrm{N}(1)-\mathrm{C}(8)$ & $124.3(3)$ \\
\hline $\mathrm{C}(3)-\mathrm{N}(2)-\mathrm{O}(1)$ & $105.6(3)$ \\
\hline $\mathrm{C}(3)-\mathrm{N}(2)-\mathrm{C}(15)$ & $124.8(3)$ \\
\hline $\mathrm{O}(1)-\mathrm{N}(2)-\mathrm{C}(15)$ & $110.6(3)$ \\
\hline $\mathrm{C}(10)-\mathrm{N}(3)-\mathrm{O}(2)$ & $108.2(3)$ \\
\hline$C(10)-N(3)-C(21)$ & $132.0(3)$ \\
\hline $\mathrm{O}(2)-\mathrm{N}(3)-\mathrm{C}(21)$ & $116.0(3)$ \\
\hline $\mathrm{N}(1)-\mathrm{C}(1)-\mathrm{O}(1)$ & $123.0(3)$ \\
\hline $\mathrm{N}(1)-\mathrm{C}(1)-\mathrm{C}(2)$ & $127.4(3)$ \\
\hline $\mathrm{O}(1)-\mathrm{C}(1)-\mathrm{C}(2)$ & $109.4(3)$ \\
\hline $\mathrm{C}(3)-\mathrm{C}(2)-\mathrm{C}(7)$ & $121.3(4)$ \\
\hline $\mathrm{C}(3)-\mathrm{C}(2)-\mathrm{C}(1)$ & $106.0(3)$ \\
\hline$C(7)-C(2)-C(1)$ & $132.6(4)$ \\
\hline $\mathrm{N}(2)-\mathrm{C}(3)-\mathrm{C}(2)$ & $110.1(3)$ \\
\hline $\mathrm{N}(2)-\mathrm{C}(3)-\mathrm{C}(4)$ & $128.7(4)$ \\
\hline$C(2)-C(3)-C(4)$ & $121.1(3)$ \\
\hline$C(5)-C(4)-C(3)$ & $116.7(4)$ \\
\hline $\mathrm{C}(5)-\mathrm{C}(4)-\mathrm{H}(4)$ & $122(2)$ \\
\hline $\mathrm{C}(3)-\mathrm{C}(4)-\mathrm{H}(4)$ & $121(2)$ \\
\hline$C(4)-C(5)-C(6)$ & $122.4(4)$ \\
\hline $\mathrm{C}(4)-\mathrm{C}(5)-\mathrm{H}(5)$ & $119(2)$ \\
\hline $\mathrm{C}(6)-\mathrm{C}(5)-\mathrm{H}(5)$ & 119(2) \\
\hline$C(7)-C(6)-C(5)$ & $120.7(4)$ \\
\hline
\end{tabular}




\begin{tabular}{|c|c|}
\hline $\mathrm{C}(7)-\mathrm{C}(6)-\mathrm{H}(6)$ & $119(2)$ \\
\hline $\mathrm{C}(5)-\mathrm{C}(6)-\mathrm{H}(6)$ & $120(2)$ \\
\hline $\mathrm{C}(6)-\mathrm{C}(7)-\mathrm{C}(2)$ & $117.8(4)$ \\
\hline $\mathrm{C}(6)-\mathrm{C}(7)-\mathrm{H}(7)$ & $124.8(19)$ \\
\hline $\mathrm{C}(2)-\mathrm{C}(7)-\mathrm{H}(7)$ & 117.4(19) \\
\hline $\mathrm{N}(1)-\mathrm{C}(8)-\mathrm{O}(2)$ & $122.0(3)$ \\
\hline $\mathrm{N}(1)-\mathrm{C}(8)-\mathrm{C}(9)$ & $128.4(3)$ \\
\hline $\mathrm{O}(2)-\mathrm{C}(8)-\mathrm{C}(9)$ & $109.5(3)$ \\
\hline $\mathrm{C}(8)-\mathrm{C}(9)-\mathrm{C}(10)$ & $105.9(3)$ \\
\hline$C(8)-C(9)-C(14)$ & $132.9(4)$ \\
\hline$C(10)-C(9)-C(14)$ & $121.1(4)$ \\
\hline $\mathrm{N}(3)-\mathrm{C}(10)-\mathrm{C}(9)$ & 109.0(3) \\
\hline $\mathrm{N}(3)-\mathrm{C}(10)-\mathrm{C}(11)$ & $129.8(4)$ \\
\hline$C(9)-C(10)-C(11)$ & $121.2(4)$ \\
\hline $\mathrm{C}(12)-\mathrm{C}(11)-\mathrm{C}(10)$ & $116.4(4)$ \\
\hline $\mathrm{C}(12)-\mathrm{C}(11)-\mathrm{H}(11)$ & $122(2)$ \\
\hline $\mathrm{C}(10)-\mathrm{C}(11)-\mathrm{H}(11)$ & $122(2)$ \\
\hline $\mathrm{C}(11)-\mathrm{C}(12)-\mathrm{C}(13)$ & $122.6(4)$ \\
\hline $\mathrm{C}(11)-\mathrm{C}(12)-\mathrm{H}(12)$ & $119(2)$ \\
\hline $\mathrm{C}(13)-\mathrm{C}(12)-\mathrm{H}(12)$ & $118(2)$ \\
\hline $\mathrm{C}(14)-\mathrm{C}(13)-\mathrm{C}(12)$ & 121.1(4) \\
\hline $\mathrm{C}(14)-\mathrm{C}(13)-\mathrm{H}(13)$ & $121(2)$ \\
\hline $\mathrm{C}(12)-\mathrm{C}(13)-\mathrm{H}(13)$ & $118(2)$ \\
\hline $\mathrm{C}(13)-\mathrm{C}(14)-\mathrm{C}(9)$ & $117.6(4)$ \\
\hline $\mathrm{C}(13)-\mathrm{C}(14)-\mathrm{H}(14)$ & $123(2)$ \\
\hline $\mathrm{C}(9)-\mathrm{C}(14)-\mathrm{H}(14)$ & $119(2)$ \\
\hline $\mathrm{N}(2)-\mathrm{C}(15)-\mathrm{C}(18)$ & $109.4(3)$ \\
\hline$N(2)-C(15)-C(17)$ & $107.8(3)$ \\
\hline $\mathrm{C}(18)-\mathrm{C}(15)-\mathrm{C}(17)$ & 109.7(4) \\
\hline$N(2)-C(15)-C(16)$ & $106.0(3)$ \\
\hline $\mathrm{C}(18)-\mathrm{C}(15)-\mathrm{C}(16)$ & 114.1(3) \\
\hline $\mathrm{C}(17)-\mathrm{C}(15)-\mathrm{C}(16)$ & 109.7(3) \\
\hline
\end{tabular}




\begin{tabular}{ll}
$\mathrm{C}(19)-\mathrm{C}(16)-\mathrm{C}(20)$ & $121.1(4)$ \\
$\mathrm{C}(19)-\mathrm{C}(16)-\mathrm{C}(15)$ & $122.3(4)$ \\
$\mathrm{C}(20)-\mathrm{C}(16)-\mathrm{C}(15)$ & $116.4(3)$ \\
$\mathrm{C}(15)-\mathrm{C}(17)-\mathrm{H}(17 \mathrm{~A})$ & $110(2)$ \\
$\mathrm{C}(15)-\mathrm{C}(17)-\mathrm{H}(17 \mathrm{~B})$ & $109(2)$ \\
$\mathrm{H}(17 \mathrm{~A})-\mathrm{C}(17)-\mathrm{H}(17 \mathrm{~B})$ & $113(3)$ \\
$\mathrm{C}(15)-\mathrm{C}(17)-\mathrm{H}(17 \mathrm{C})$ & $109(2)$ \\
$\mathrm{H}(17 \mathrm{~A})-\mathrm{C}(17)-\mathrm{H}(17 \mathrm{C})$ & $110(3)$ \\
$\mathrm{H}(17 \mathrm{~B})-\mathrm{C}(17)-\mathrm{H}(17 \mathrm{C})$ & $105(3)$ \\
$\mathrm{C}(15)-\mathrm{C}(18)-\mathrm{H}(18 \mathrm{~A})$ & $116(2)$ \\
$\mathrm{C}(15)-\mathrm{C}(18)-\mathrm{H}(18 \mathrm{~B})$ & $110(3)$ \\
$\mathrm{H}(18 \mathrm{~A})-\mathrm{C}(18)-\mathrm{H}(18 \mathrm{~B})$ & $111(3)$ \\
$\mathrm{C}(15)-\mathrm{C}(18)-\mathrm{H}(18 \mathrm{C})$ & $107(2)$ \\
$\mathrm{H}(18 \mathrm{~A})-\mathrm{C}(18)-\mathrm{H}(18 \mathrm{C})$ & $108(3)$ \\
$\mathrm{H}(18 \mathrm{~B})-\mathrm{C}(18)-\mathrm{H}(18 \mathrm{C})$ & $105(3)$ \\
$\mathrm{C}(16)-\mathrm{C}(19)-\mathrm{H}(19 \mathrm{~A})$ & $125(3)$ \\
$\mathrm{C}(16)-\mathrm{C}(19)-\mathrm{H}(19 \mathrm{~B})$ & $120(2)$ \\
$\mathrm{H}(19 \mathrm{~A})-\mathrm{C}(19)-\mathrm{H}(19 \mathrm{~B})$ & $115(3)$ \\
$\mathrm{C}(16)-\mathrm{C}(20)-\mathrm{H}(20 \mathrm{~A})$ & $110(3)$ \\
$\mathrm{C}(16)-\mathrm{C}(20)-\mathrm{H}(20 \mathrm{~B})$ & $113(2)$ \\
$\mathrm{H}(20 \mathrm{~A})-\mathrm{C}(20)-\mathrm{H}(20 \mathrm{~B})$ & $104(3)$ \\
$\mathrm{C}(16)-\mathrm{C}(20)-\mathrm{H}(20 \mathrm{C})$ & $115(3)$ \\
$\mathrm{H}(20 \mathrm{~A})-\mathrm{C}(20)-\mathrm{H}(20 \mathrm{C})$ & $113(4)$ \\
$\mathrm{H}(20 \mathrm{~B})-\mathrm{C}(20)-\mathrm{H}(20 \mathrm{C})$ & $101(3)$ \\
$\mathrm{N}(3)-\mathrm{C}(21)-\mathrm{C}(22)$ & $105.3(3)$ \\
$\mathrm{N}(3)-\mathrm{C}(21)-\mathrm{C}(24)$ & $108.8(3)$ \\
$\mathrm{C}(22)-\mathrm{C}(21)-\mathrm{C}(24)$ & $114.3(4)$ \\
$\mathrm{N}(3)-\mathrm{C}(21)-\mathrm{C}(23)$ & $108.1(3)$ \\
$\mathrm{C}(22)-\mathrm{C}(21)-\mathrm{C}(23)$ & $110.4(4)$ \\
$\mathrm{C}(24)-\mathrm{C}(21)-\mathrm{C}(23)$ & $109.5(4)$ \\
$\mathrm{C}(22)-\mathrm{C}(21)$ & $120.9(5)$ \\
\hline
\end{tabular}




\begin{tabular}{ll}
$\mathrm{C}(26)-\mathrm{C}(22)-\mathrm{C}(21)$ & $116.6(4)$ \\
$\mathrm{C}(21)-\mathrm{C}(23)-\mathrm{H}(23 \mathrm{~A})$ & $111(3)$ \\
$\mathrm{C}(21)-\mathrm{C}(23)-\mathrm{H}(23 \mathrm{~B})$ & $108(2)$ \\
$\mathrm{H}(23 \mathrm{~A})-\mathrm{C}(23)-\mathrm{H}(23 \mathrm{~B})$ & $108(3)$ \\
$\mathrm{C}(21)-\mathrm{C}(23)-\mathrm{H}(23 \mathrm{C})$ & $111(2)$ \\
$\mathrm{H}(23 \mathrm{~A})-\mathrm{C}(23)-\mathrm{H}(23 \mathrm{C})$ & $103(3)$ \\
$\mathrm{H}(23 \mathrm{~B})-\mathrm{C}(23)-\mathrm{H}(23 \mathrm{C})$ & $115(3)$ \\
$\mathrm{C}(21)-\mathrm{C}(24)-\mathrm{H}(24 \mathrm{~A})$ & $111(3)$ \\
$\mathrm{C}(21)-\mathrm{C}(24)-\mathrm{H}(24 \mathrm{~B})$ & $108(2)$ \\
$\mathrm{H}(24 \mathrm{~A})-\mathrm{C}(24)-\mathrm{H}(24 \mathrm{~B})$ & $114(3)$ \\
$\mathrm{C}(21)-\mathrm{C}(24)-\mathrm{H}(24 \mathrm{C})$ & $112(2)$ \\
$\mathrm{H}(24 \mathrm{~A})-\mathrm{C}(24)-\mathrm{H}(24 \mathrm{C})$ & $107(3)$ \\
$\mathrm{H}(24 \mathrm{~B})-\mathrm{C}(24)-\mathrm{H}(24 \mathrm{C})$ & $104(3)$ \\
$\mathrm{C}(22)-\mathrm{C}(25)-\mathrm{H}(25 \mathrm{~A})$ & $117(2)$ \\
$\mathrm{C}(22)-\mathrm{C}(25)-\mathrm{H}(25 \mathrm{~B})$ & $123(2)$ \\
$\mathrm{H}(25 \mathrm{~A})-\mathrm{C}(25)-\mathrm{H}(25 \mathrm{~B})$ & $120(3)$ \\
$\mathrm{C}(22)-\mathrm{C}(26)-\mathrm{H}(26 \mathrm{~A})$ & $110(3)$ \\
$\mathrm{C}(22)-\mathrm{C}(26)-\mathrm{H}(26 \mathrm{~B})$ & $110(3)$ \\
$\mathrm{H}(26 \mathrm{~A})-\mathrm{C}(26)-\mathrm{H}(26 \mathrm{~B})$ & $114(4)$ \\
$\mathrm{C}(22)-\mathrm{C}(26)-\mathrm{H}(26 \mathrm{C})$ & $110(2)$ \\
$\mathrm{H}(26 \mathrm{~A})-\mathrm{C}(26)-\mathrm{H}(26 \mathrm{C})$ & $101(4)$ \\
$\mathrm{H}(26 \mathrm{~B})-\mathrm{C}(26)-\mathrm{H}(26 \mathrm{C})$ & $111(3)$ \\
$\mathrm{F}(3)-\mathrm{B}(1)-\mathrm{F}(1)$ & $112.3(5)$ \\
$\mathrm{F}(3)-\mathrm{B}(1)-\mathrm{F}(2)$ & $109.1(4)$ \\
$\mathrm{F}(1)-\mathrm{B}(1)-\mathrm{F}(2)$ & $107.5(4)$ \\
$\mathrm{F}(3)-\mathrm{B}(1)-\mathrm{F}(4)$ & $109.1(4)$ \\
$\mathrm{F}(1)-\mathrm{B}(1)-\mathrm{F}(4)$ & $109.2(4)$ \\
& $109.6(5)$ \\
\hline
\end{tabular}

Symmetry transformations used to generate equivalent atoms: 
Table 4. Anisotropic displacement parameters $\left(\AA^{2} \times 10^{3}\right)$ for 9a. The anisotropic displacement factor exponent takes the form: $-2 \pi^{2}\left[h^{2} a^{* 2} U^{11}+\ldots+2 h k a^{*} b^{*} U^{12}\right]$

\begin{tabular}{|c|c|c|c|c|c|c|}
\hline & $\mathrm{U}^{11}$ & $\mathrm{U}^{22}$ & $\mathrm{U}^{33}$ & $\mathrm{U}^{23}$ & $\mathrm{U}^{13}$ & $\mathrm{U}^{12}$ \\
\hline $\mathrm{O}(1)$ & $26(1)$ & $39(2)$ & $19(1)$ & $5(1)$ & $-2(1)$ & $-8(1)$ \\
\hline $\mathrm{O}(2)$ & $27(1)$ & $35(2)$ & 19(1) & $5(1)$ & $-3(1)$ & $-8(1)$ \\
\hline $\mathrm{N}(1)$ & $24(2)$ & $36(2)$ & $20(2)$ & 2(2) & $2(1)$ & $-6(2)$ \\
\hline $\mathrm{N}(2)$ & $22(2)$ & $34(2)$ & $21(2)$ & $4(2)$ & $-4(1)$ & $-8(2)$ \\
\hline $\mathrm{N}(3)$ & $40(2)$ & $52(3)$ & $22(2)$ & $11(2)$ & $-3(2)$ & $-23(2)$ \\
\hline $\mathrm{C}(1)$ & $19(2)$ & $41(3)$ & $19(2)$ & $-3(2)$ & $0(2)$ & $1(2)$ \\
\hline$C(2)$ & $20(2)$ & $32(3)$ & $17(2)$ & $0(2)$ & $1(2)$ & $-1(2)$ \\
\hline $\mathrm{C}(3)$ & $17(2)$ & $38(3)$ & $16(2)$ & $2(2)$ & $-1(2)$ & $7(2)$ \\
\hline $\mathrm{C}(4)$ & $21(2)$ & $34(3)$ & $23(2)$ & $-2(2)$ & $-1(2)$ & $-4(2)$ \\
\hline $\mathrm{C}(5)$ & $25(2)$ & $35(3)$ & $29(2)$ & $7(2)$ & $5(2)$ & $-3(2)$ \\
\hline $\mathrm{C}(6)$ & $32(2)$ & $48(3)$ & $21(2)$ & $9(2)$ & $2(2)$ & $2(2)$ \\
\hline $\mathrm{C}(7)$ & $29(2)$ & $41(3)$ & $21(2)$ & $-4(2)$ & $-1(2)$ & $-4(2)$ \\
\hline $\mathrm{C}(8)$ & $26(2)$ & $34(3)$ & $17(2)$ & $-6(2)$ & $-1(2)$ & $2(2)$ \\
\hline $\mathrm{C}(9)$ & $19(2)$ & $33(3)$ & $19(2)$ & $2(2)$ & $2(2)$ & $1(2)$ \\
\hline $\mathrm{C}(10)$ & $28(2)$ & $35(3)$ & $21(2)$ & $-3(2)$ & $-1(2)$ & $-3(2)$ \\
\hline $\mathrm{C}(11)$ & $34(2)$ & $45(3)$ & $30(3)$ & $9(2)$ & $0(2)$ & $-11(2)$ \\
\hline $\mathrm{C}(12)$ & $35(3)$ & $49(3)$ & $32(3)$ & $1(2)$ & $-3(2)$ & $-16(2)$ \\
\hline $\mathrm{C}(13)$ & $37(3)$ & $41(3)$ & $27(2)$ & $-3(2)$ & $-7(2)$ & $-9(2)$ \\
\hline $\mathrm{C}(14)$ & $32(2)$ & $34(3)$ & $24(2)$ & $0(2)$ & $-5(2)$ & $-2(2)$ \\
\hline$C(15)$ & $23(2)$ & $30(3)$ & $25(2)$ & $4(2)$ & $-4(2)$ & $-1(2)$ \\
\hline$C(16)$ & $29(2)$ & $33(3)$ & $16(2)$ & $0(2)$ & $-6(2)$ & $-3(2)$ \\
\hline $\mathrm{C}(17)$ & $33(3)$ & $46(4)$ & $26(2)$ & $6(2)$ & $-5(2)$ & $-8(2)$ \\
\hline$C(18)$ & $31(3)$ & $39(3)$ & $36(3)$ & $-3(2)$ & $-4(2)$ & $3(2)$ \\
\hline $\mathrm{C}(19)$ & $33(3)$ & $42(4)$ & $34(3)$ & $-2(2)$ & $0(2)$ & $-10(2)$ \\
\hline $\mathrm{C}(20)$ & $31(2)$ & $43(4)$ & $32(3)$ & $-4(2)$ & $-5(2)$ & $2(2)$ \\
\hline $\mathrm{C}(21)$ & $33(2)$ & $42(3)$ & $16(2)$ & $7(2)$ & $-2(2)$ & $0(2)$ \\
\hline
\end{tabular}




\begin{tabular}{lllllll}
$\mathrm{C}(22)$ & $34(2)$ & $32(3)$ & $21(2)$ & $3(2)$ & $-4(2)$ & $1(2)$ \\
$\mathrm{C}(23)$ & $40(3)$ & $53(4)$ & $21(3)$ & $0(2)$ & $-6(2)$ & $-14(3)$ \\
$\mathrm{C}(24)$ & $42(3)$ & $36(4)$ & $40(3)$ & $2(2)$ & $2(2)$ & $6(2)$ \\
$\mathrm{C}(25)$ & $37(3)$ & $51(4)$ & $39(3)$ & $9(3)$ & $6(2)$ & $-5(3)$ \\
$\mathrm{C}(26)$ & $47(3)$ & $45(4)$ & $31(3)$ & $-9(3)$ & $3(2)$ & $7(3)$ \\
$\mathrm{B}(1)$ & $27(3)$ & $56(4)$ & $32(3)$ & $6(3)$ & $-2(2)$ & $1(3)$ \\
$\mathrm{F}(1)$ & $82(2)$ & $82(3)$ & $130(3)$ & $-36(2)$ & $49(2)$ & $-29(2)$ \\
$\mathrm{F}(2)$ & $132(3)$ & $107(3)$ & $45(2)$ & $24(2)$ & $5(2)$ & $-5(2)$ \\
$\mathrm{F}(3)$ & $67(2)$ & $128(3)$ & $75(2)$ & $-4(2)$ & $-24(2)$ & $39(2)$ \\
$\mathrm{F}(4)$ & $63(2)$ & $60(2)$ & $72(2)$ & $-13(2)$ & $4(2)$ & $8(2)$ \\
\hline
\end{tabular}


Table 5. Hydrogen coordinates $\left(x 10^{4}\right)$ and isotropic displacement parameters $\left(\AA^{2} \times 10^{3}\right)$ for 9a.

\begin{tabular}{|c|c|c|c|c|}
\hline & $\mathrm{x}$ & $\mathrm{y}$ & $\mathrm{z}$ & $\mathrm{U}(\mathrm{eq})$ \\
\hline $\mathrm{H}(4)$ & $6860(30)$ & $3550(30)$ & $5039(15)$ & $18(9)$ \\
\hline $\mathrm{H}(5)$ & $7290(30)$ & $3130(40)$ & $6108(16)$ & $30(11)$ \\
\hline $\mathrm{H}(6)$ & $8770(30)$ & $4340(30)$ & $6711(17)$ & $28(10)$ \\
\hline $\mathrm{H}(7)$ & $9710(30)$ & $6130(30)$ & $6252(14)$ & $16(9)$ \\
\hline $\mathrm{H}(11)$ & $12620(30)$ & $11050(30)$ & $3699(16)$ & $23(10)$ \\
\hline $\mathrm{H}(12)$ & $14090(30)$ & $11430(30)$ & $4538(15)$ & $30(11)$ \\
\hline $\mathrm{H}(13)$ & $13870(30)$ & $10600(30)$ & $5535(16)$ & $29(10)$ \\
\hline $\mathrm{H}(14)$ & $12210(40)$ & $9160(40)$ & $5683(18)$ & $46(13)$ \\
\hline $\mathrm{H}(17 \mathrm{~A})$ & $7870(40)$ & $5720(40)$ & $3199(18)$ & $48(13)$ \\
\hline $\mathrm{H}(17 \mathrm{~B})$ & $6360(40)$ & $6250(40)$ & $3093(17)$ & $42(12)$ \\
\hline $\mathrm{H}(17 \mathrm{C})$ & $7380(30)$ & $7100(40)$ & $3478(17)$ & $43(13)$ \\
\hline $\mathrm{H}(18 \mathrm{~A})$ & $5100(40)$ & $6600(40)$ & $4050(18)$ & $44(12)$ \\
\hline $\mathrm{H}(18 \mathrm{~B})$ & $5620(40)$ & $5980(40)$ & $4690(20)$ & $51(14)$ \\
\hline $\mathrm{H}(18 \mathrm{C})$ & $6310(30)$ & $7170(40)$ & $4448(16)$ & $34(11)$ \\
\hline $\mathrm{H}(19 \mathrm{~A})$ & $4500(40)$ & $4430(40)$ & $4021(18)$ & $42(13)$ \\
\hline $\mathrm{H}(19 \mathrm{~B})$ & $4890(30)$ & $3080(40)$ & $3753(17)$ & $40(13)$ \\
\hline $\mathrm{H}(20 \mathrm{~A})$ & $6870(50)$ & $2470(50)$ & $3540(20)$ & $94(19)$ \\
\hline $\mathrm{H}(20 \mathrm{~B})$ & $7960(30)$ & $3150(30)$ & $3983(17)$ & $27(10)$ \\
\hline $\mathrm{H}(20 \mathrm{C})$ & $7810(40)$ & $3670(40)$ & $3320(20)$ & $57(15)$ \\
\hline $\mathrm{H}(23 \mathrm{~A})$ & $9120(40)$ & $8710(40)$ & $2450(20)$ & $64(15)$ \\
\hline $\mathrm{H}(23 \mathrm{~B})$ & $9430(40)$ & $7740(50)$ & $3014(19)$ & $63(16)$ \\
\hline $\mathrm{H}(23 \mathrm{C})$ & $8560(40)$ & $9100(40)$ & $3082(18)$ & $45(13)$ \\
\hline $\mathrm{H}(24 \mathrm{~A})$ & $12490(40)$ & $9080(40)$ & $2960(20)$ & $65(15)$ \\
\hline $\mathrm{H}(24 \mathrm{~B})$ & $11620(40)$ & $7750(50)$ & 3003(19) & $59(15)$ \\
\hline $\mathrm{H}(24 \mathrm{C})$ & 11560(40) & $8530(40)$ & $2360(20)$ & $49(13)$ \\
\hline $\mathrm{H}(25 \mathrm{~A})$ & $11040(40)$ & $12070(50)$ & $2360(20)$ & $61(16)$ \\
\hline $\mathrm{H}(25 \mathrm{~B})$ & $11820(30)$ & $10690(40)$ & $2290(17)$ & $33(12)$ \\
\hline $\mathrm{H}(26 \mathrm{~A})$ & $9450(40)$ & $12240(50)$ & $3000(20)$ & $76(19)$ \\
\hline $\mathrm{H}(26 \mathrm{~B})$ & $8570(50)$ & 10960(50) & $3150(20)$ & $84(17)$ \\
\hline $\mathrm{H}(26 \mathrm{C})$ & $9750(40)$ & $11670(40)$ & $3620(20)$ & $56(14)$ \\
\hline
\end{tabular}


6, ${ }^{1} \mathrm{H}$ NMR, $300 \mathrm{MHz}, \mathrm{CDCl}_{3}$

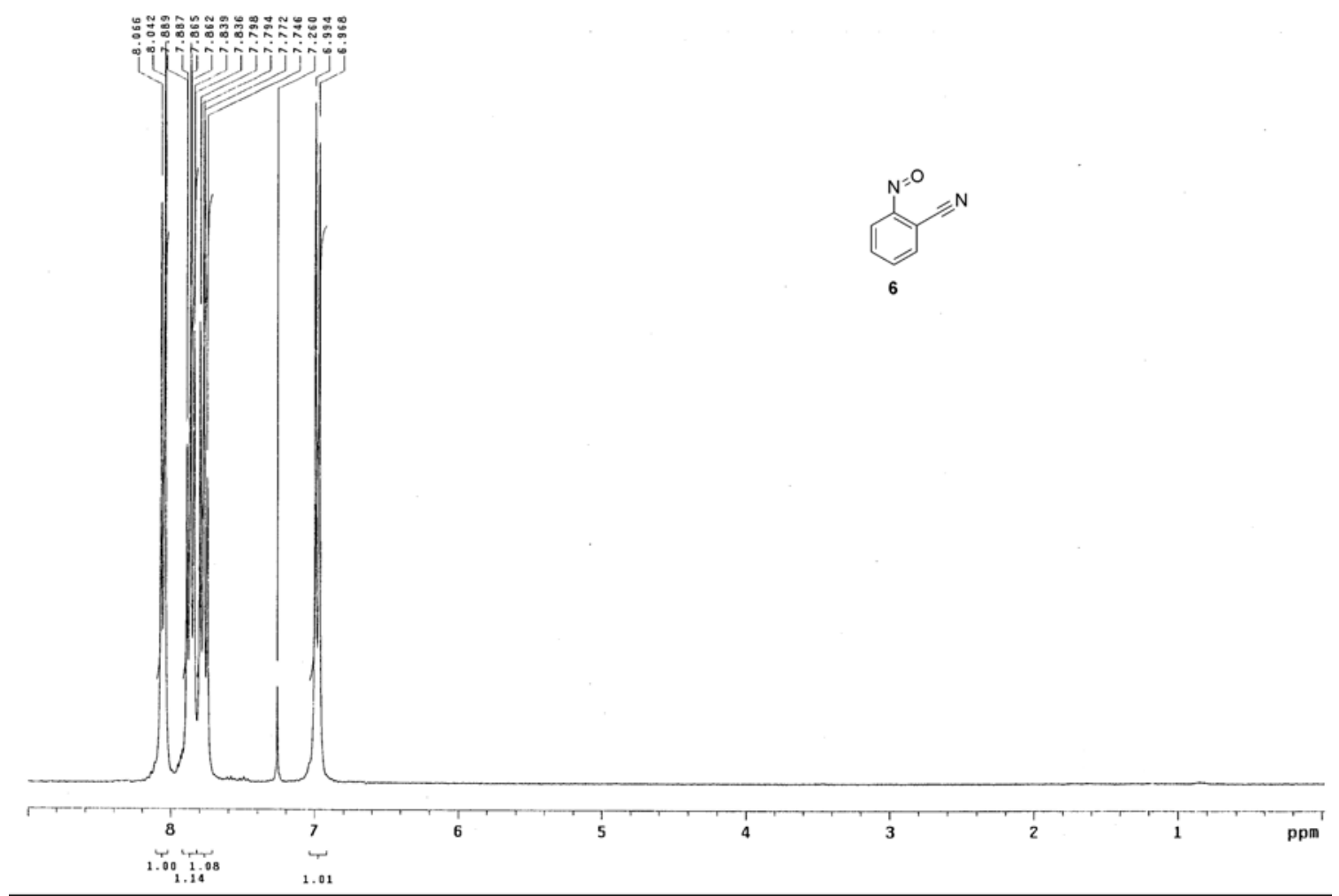

6, ${ }^{13} \mathrm{C} \mathrm{NMR}, 75 \mathrm{MHz}, \mathrm{CDCl}_{3}$

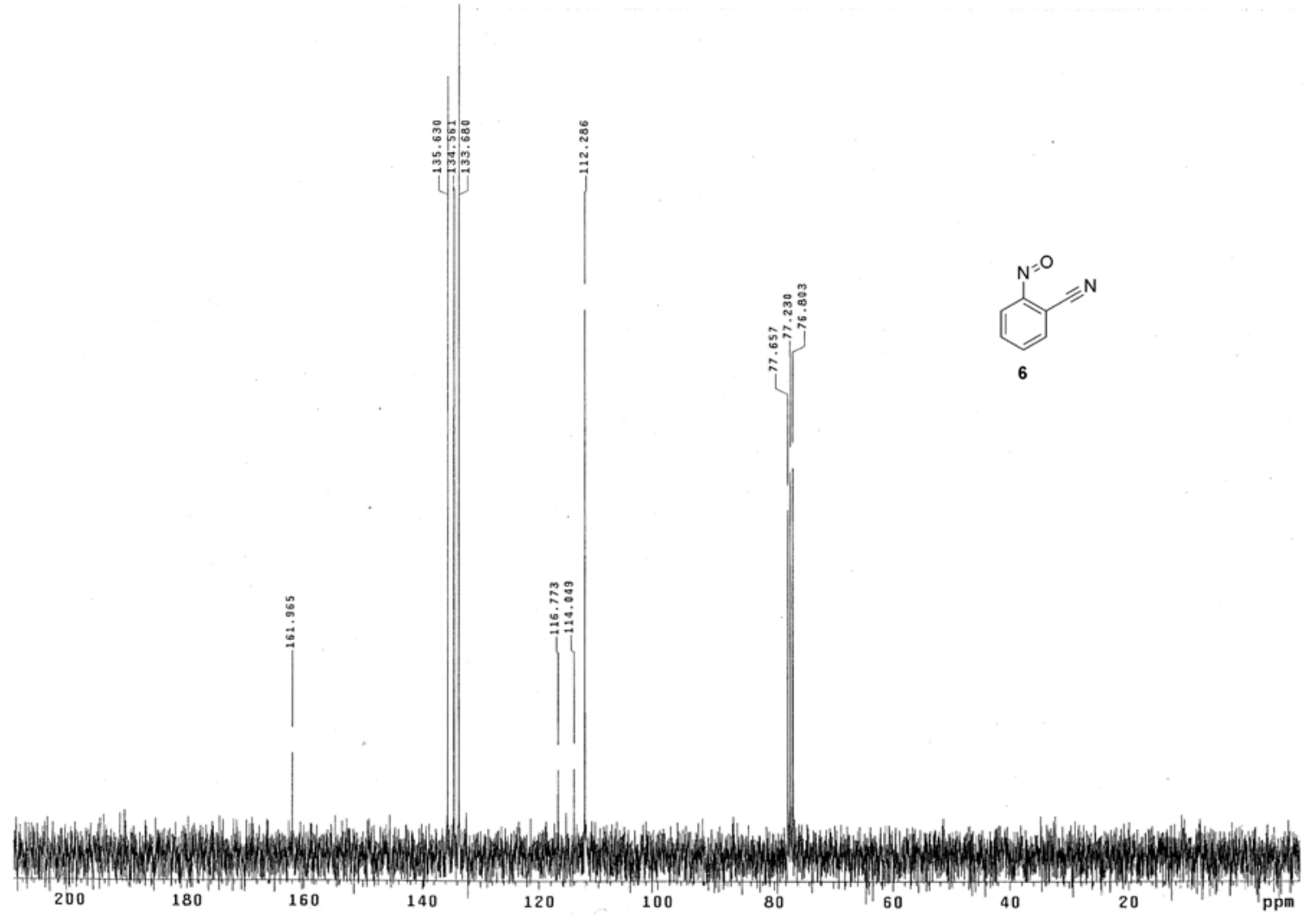


7a, ${ }^{1} \mathrm{H}$ NMR, $300 \mathrm{MHz}, \mathrm{CDCl}_{3}$

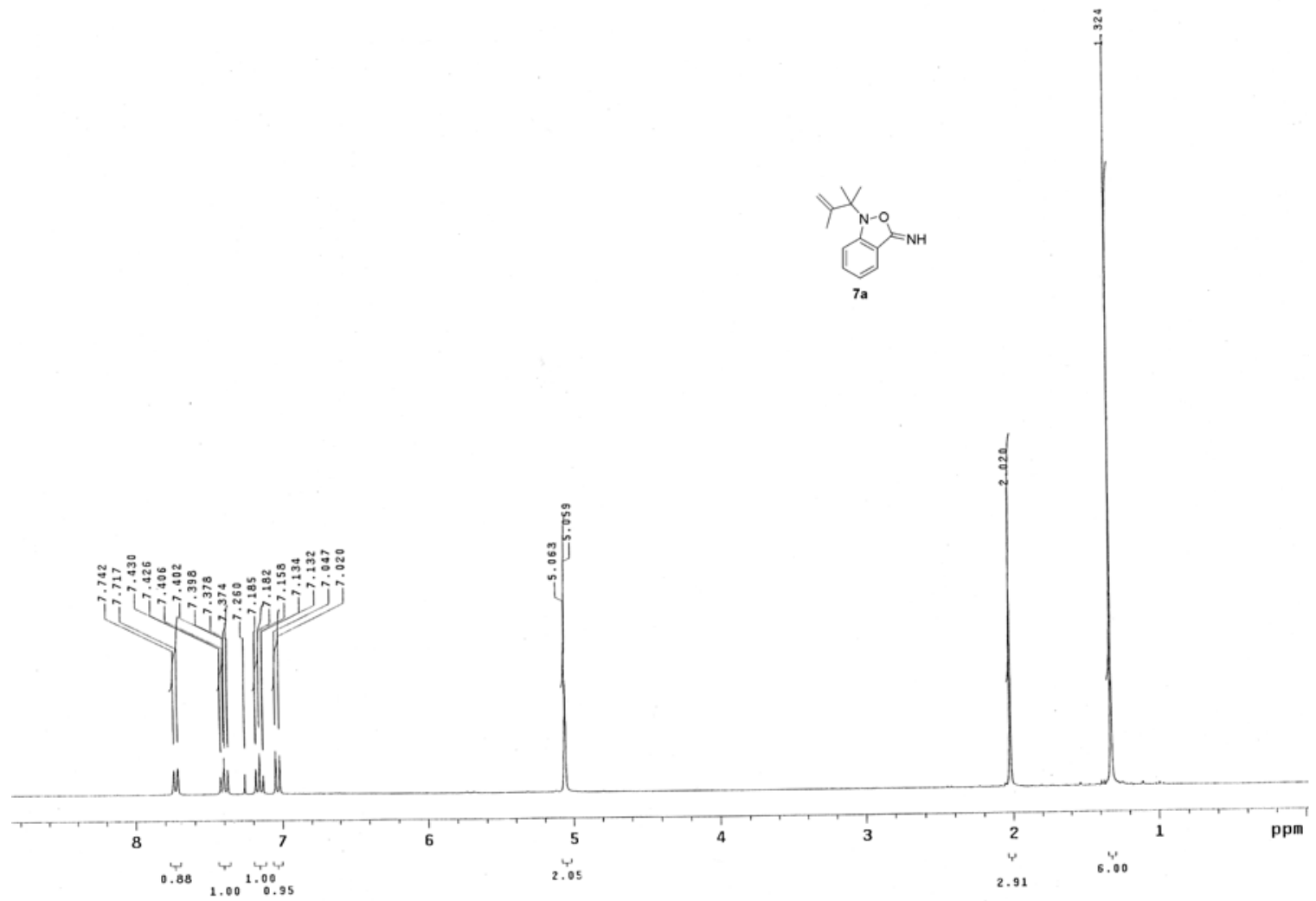

7a, ${ }^{13} \mathrm{C}$ NMR, $75 \mathrm{MHz}, \mathrm{CDCl}_{3}$

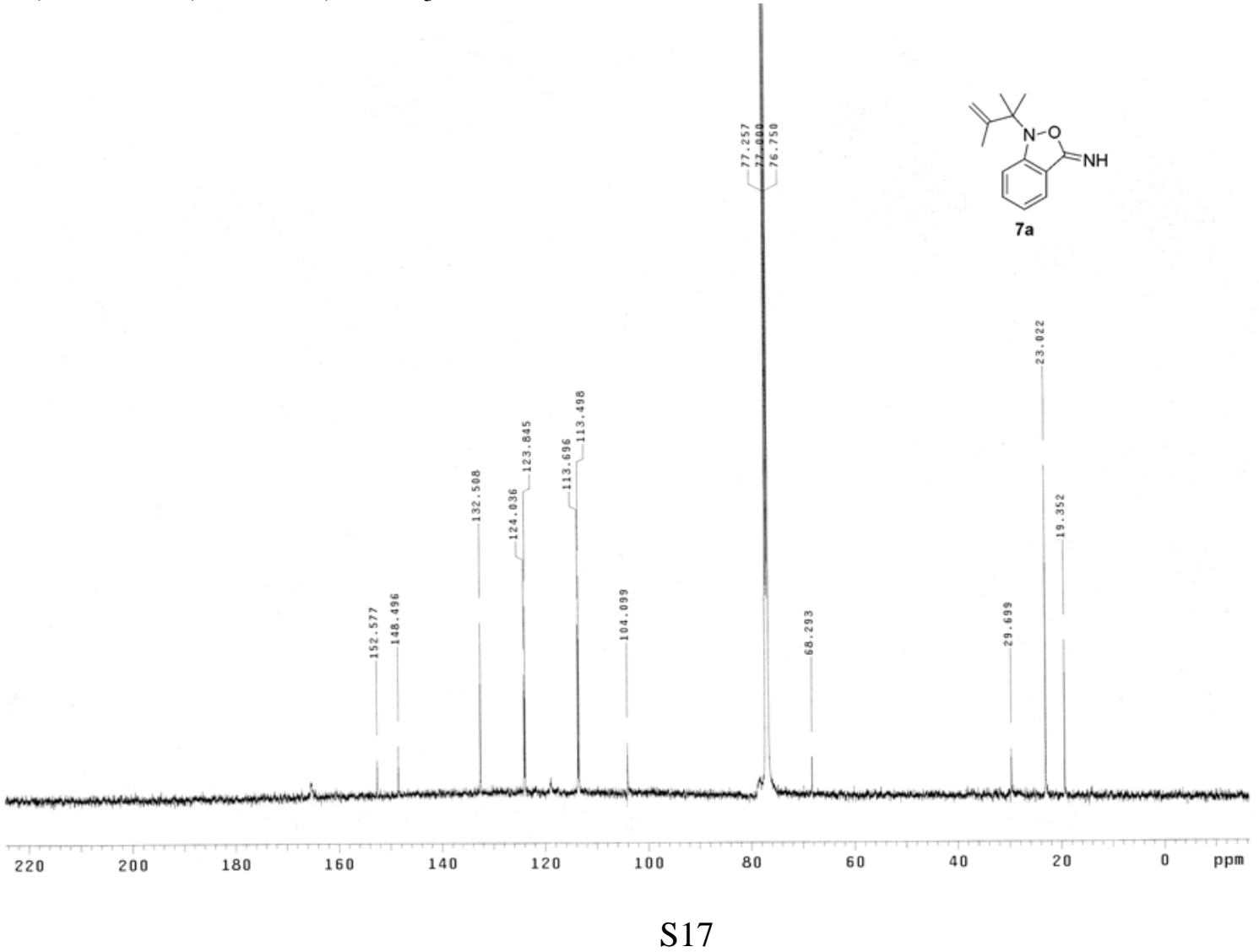


7b, ${ }^{1} \mathrm{H}$ NMR, $300 \mathrm{MHz}, \mathrm{CDCl}_{3}$

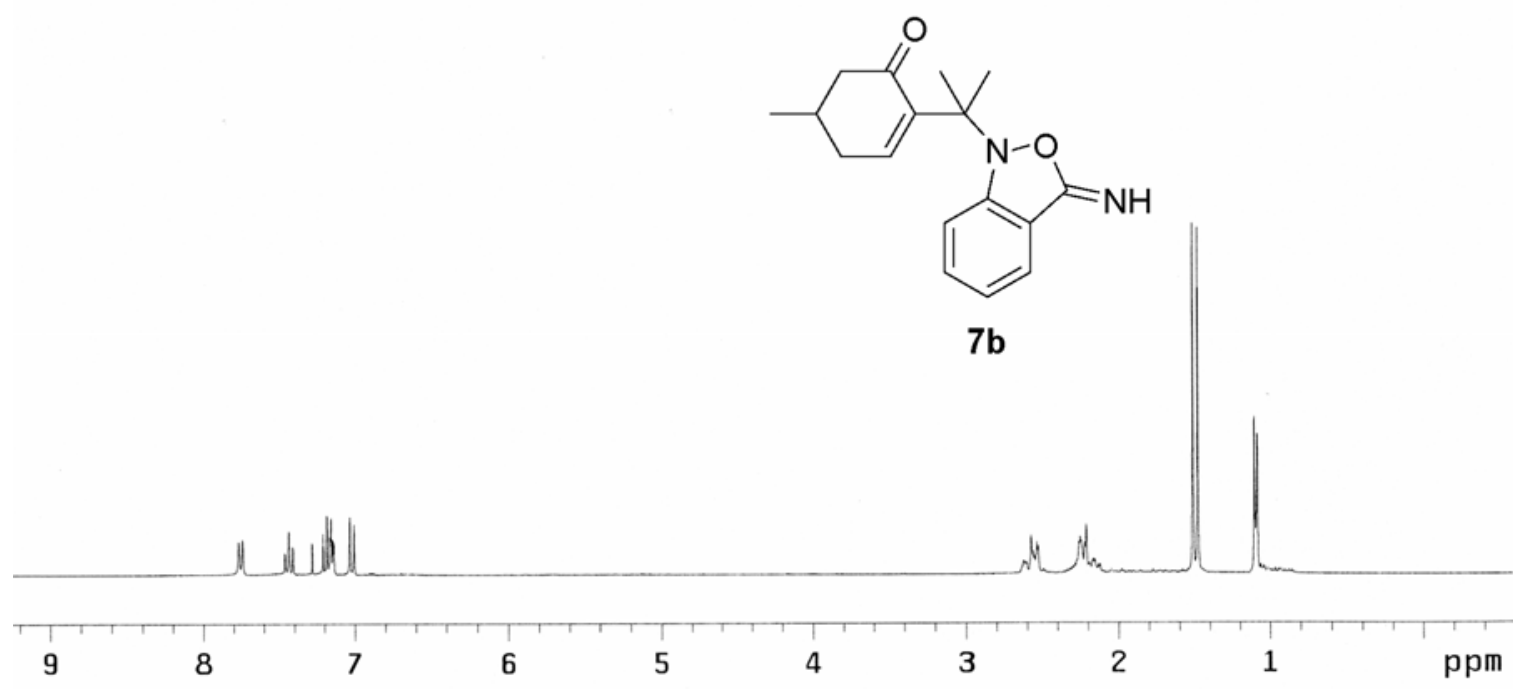

7b, ${ }^{13} \mathrm{C}$ NMR, $75 \mathrm{MHz}, \mathrm{CDCl}_{3}$

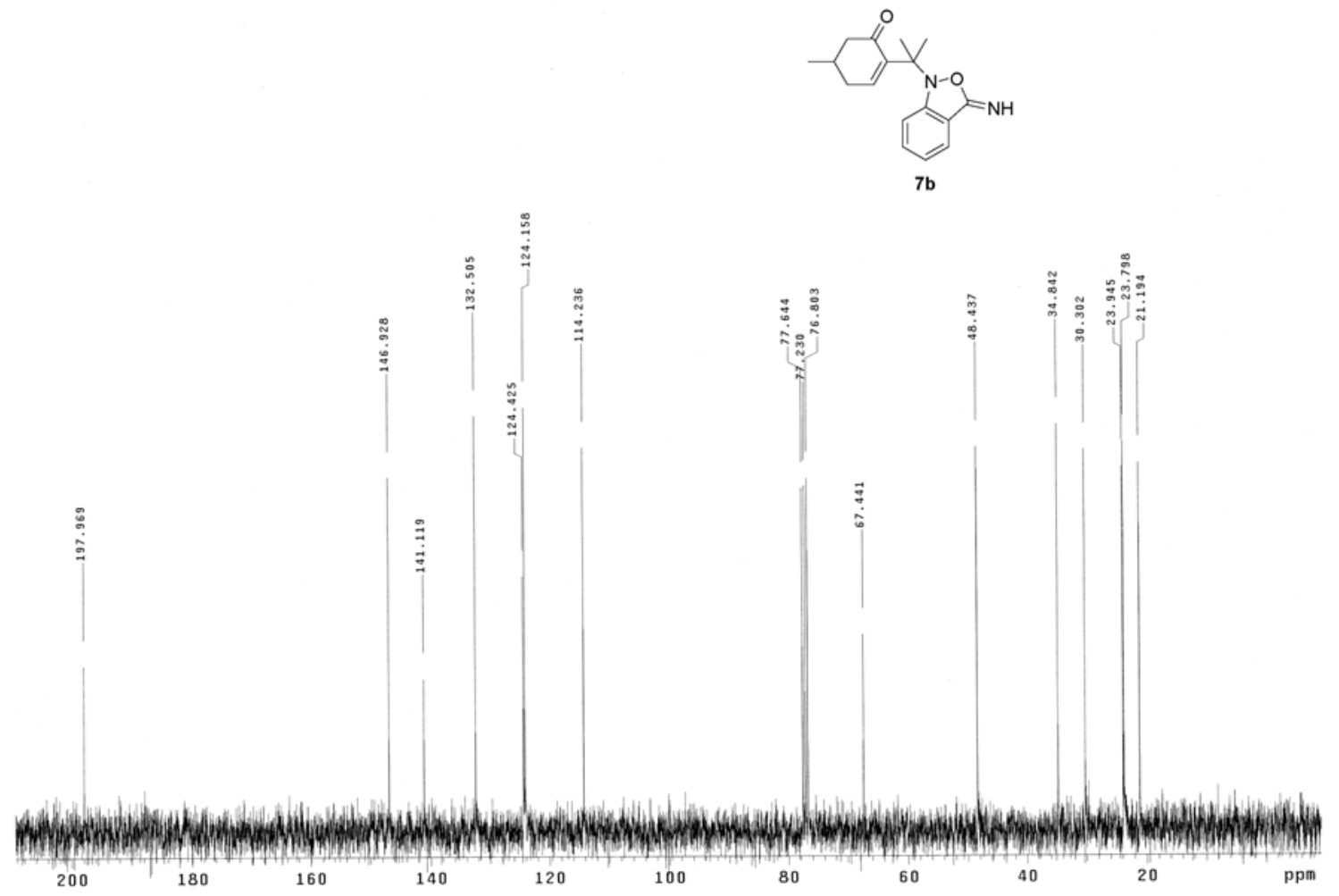


7c, ${ }^{1} \mathrm{H}$ NMR, $300 \mathrm{MHz}, \mathrm{CDCl}_{3}$
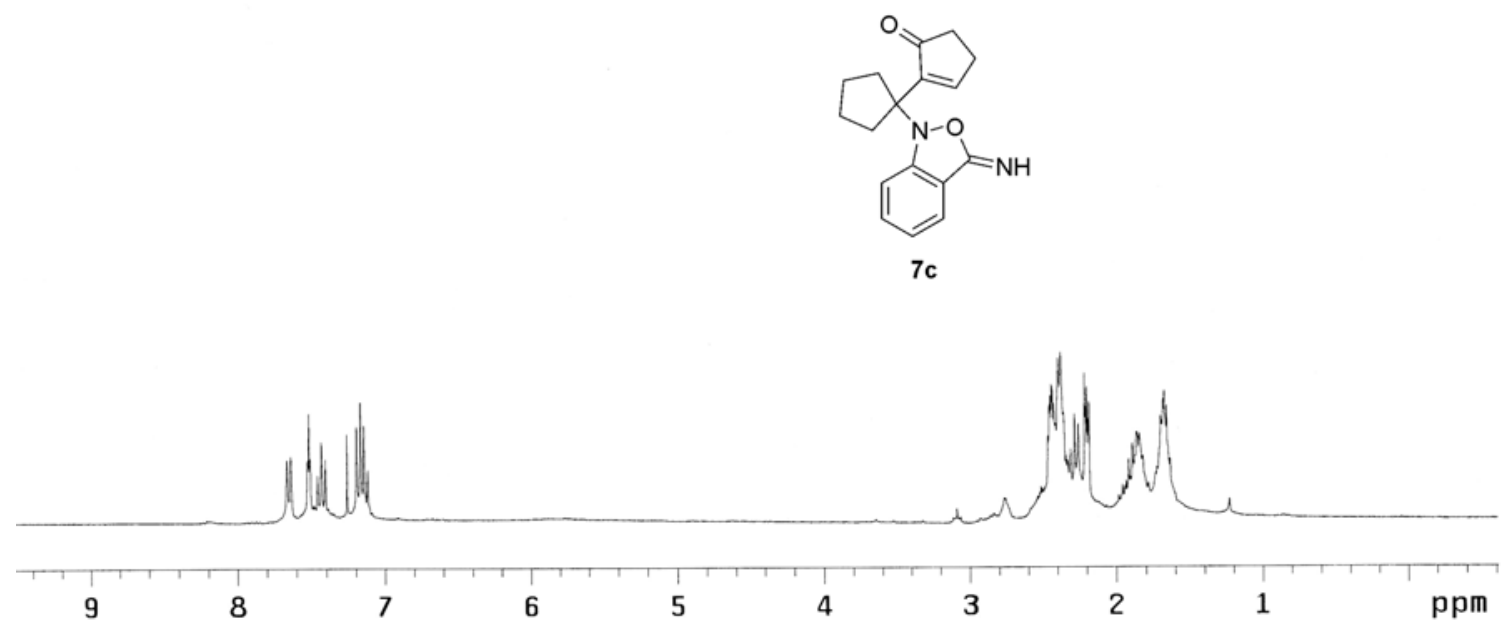

7c, ${ }^{13} \mathrm{C}$ NMR, $75 \mathrm{MHz}, \mathrm{CDCl}_{3}$

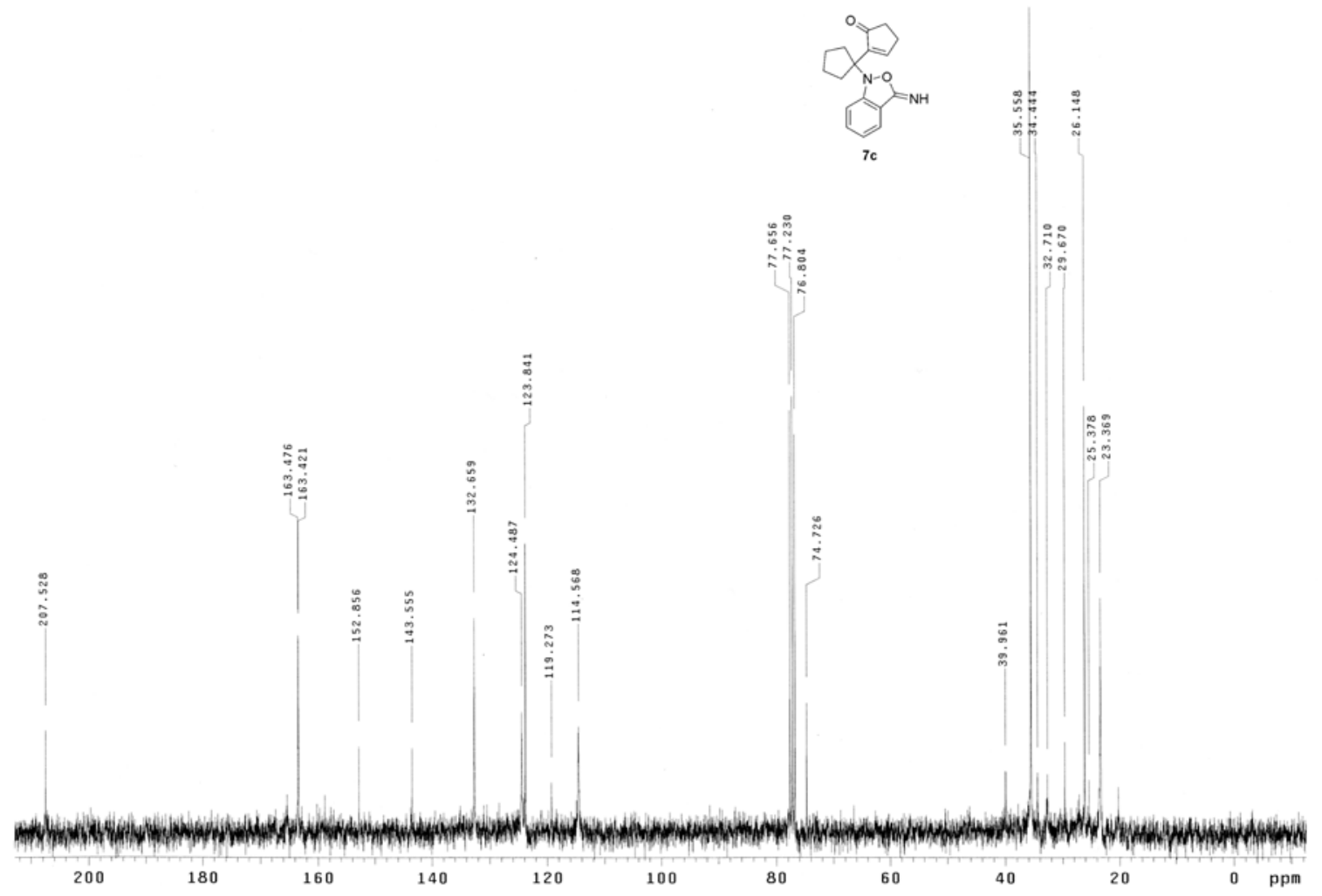


7d, ${ }^{1} \mathrm{H}$ NMR, $300 \mathrm{MHz}, \mathrm{CDCl}_{3}$
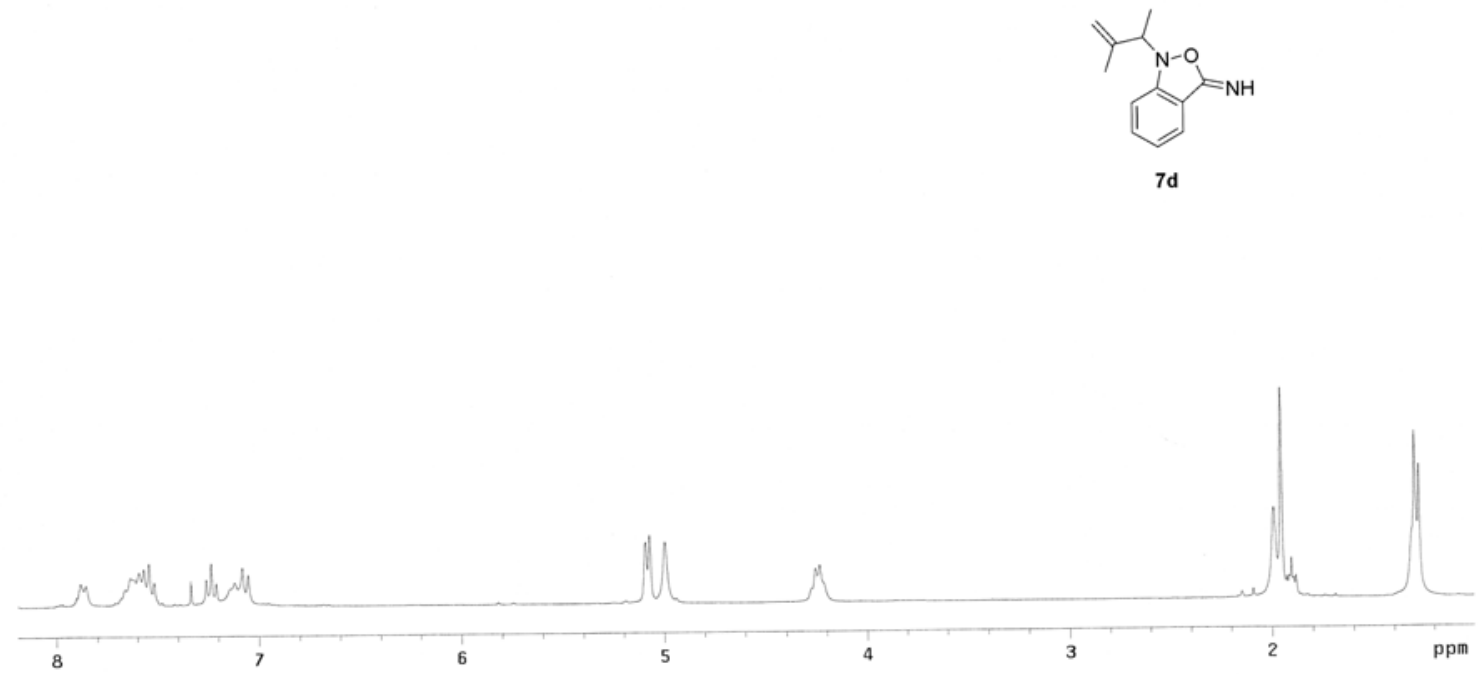

7d, ${ }^{13} \mathrm{C}$ NMR, $75 \mathrm{MHz}, \mathrm{CDCl}_{3}$

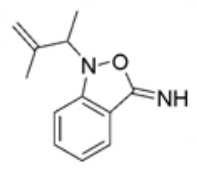

7d

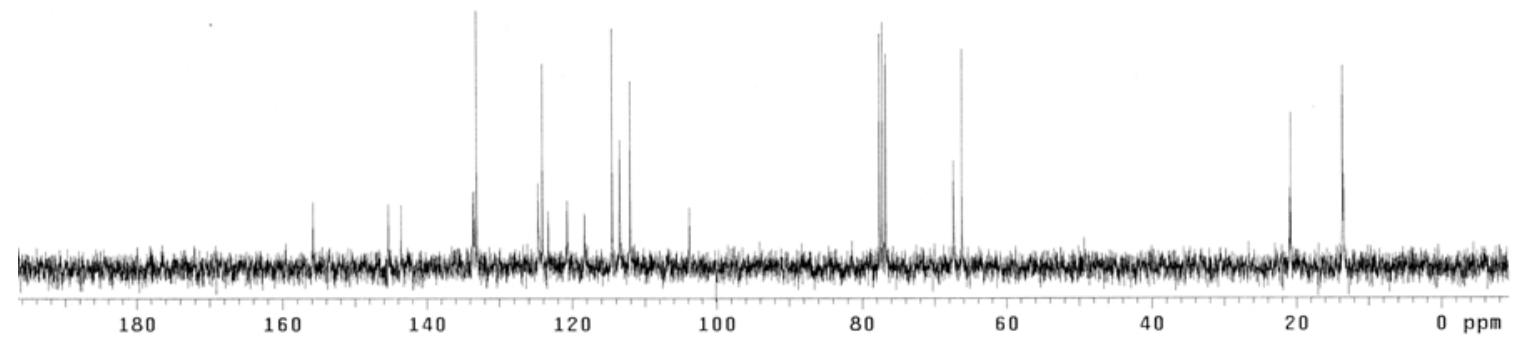


7e, ${ }^{1} \mathrm{H}$ NMR, $300 \mathrm{MHz}, \mathrm{CDCl}_{3}$

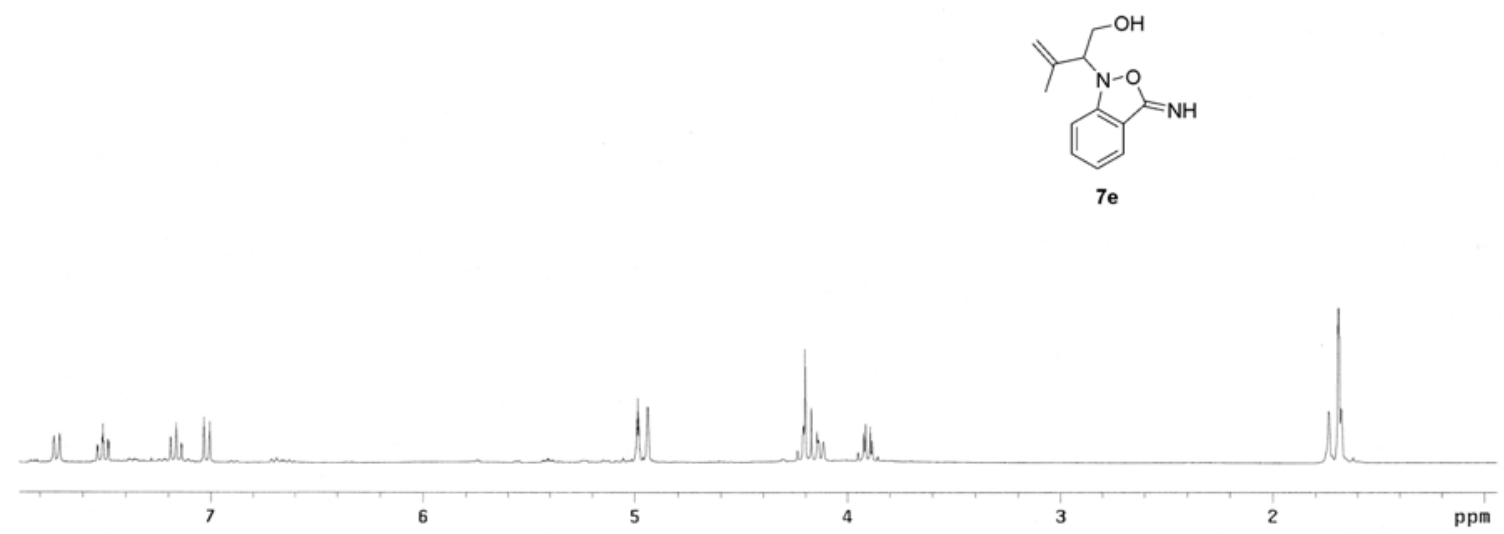

7e, ${ }^{13} \mathrm{C}$ NMR, $75 \mathrm{MHz}, \mathrm{CDCl}_{3}$
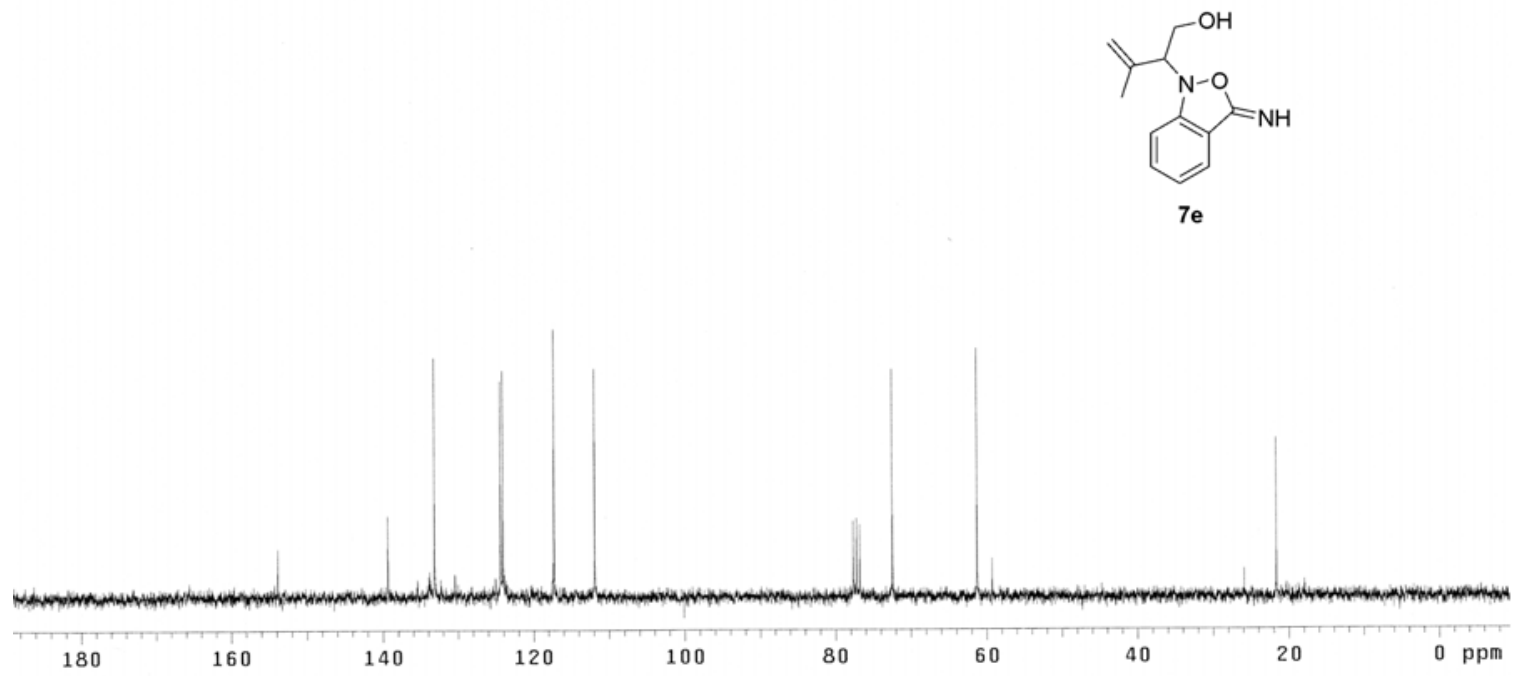
8, ${ }^{1} \mathrm{H}$ NMR, $300 \mathrm{MHz}, \mathrm{CDCl}_{3}$

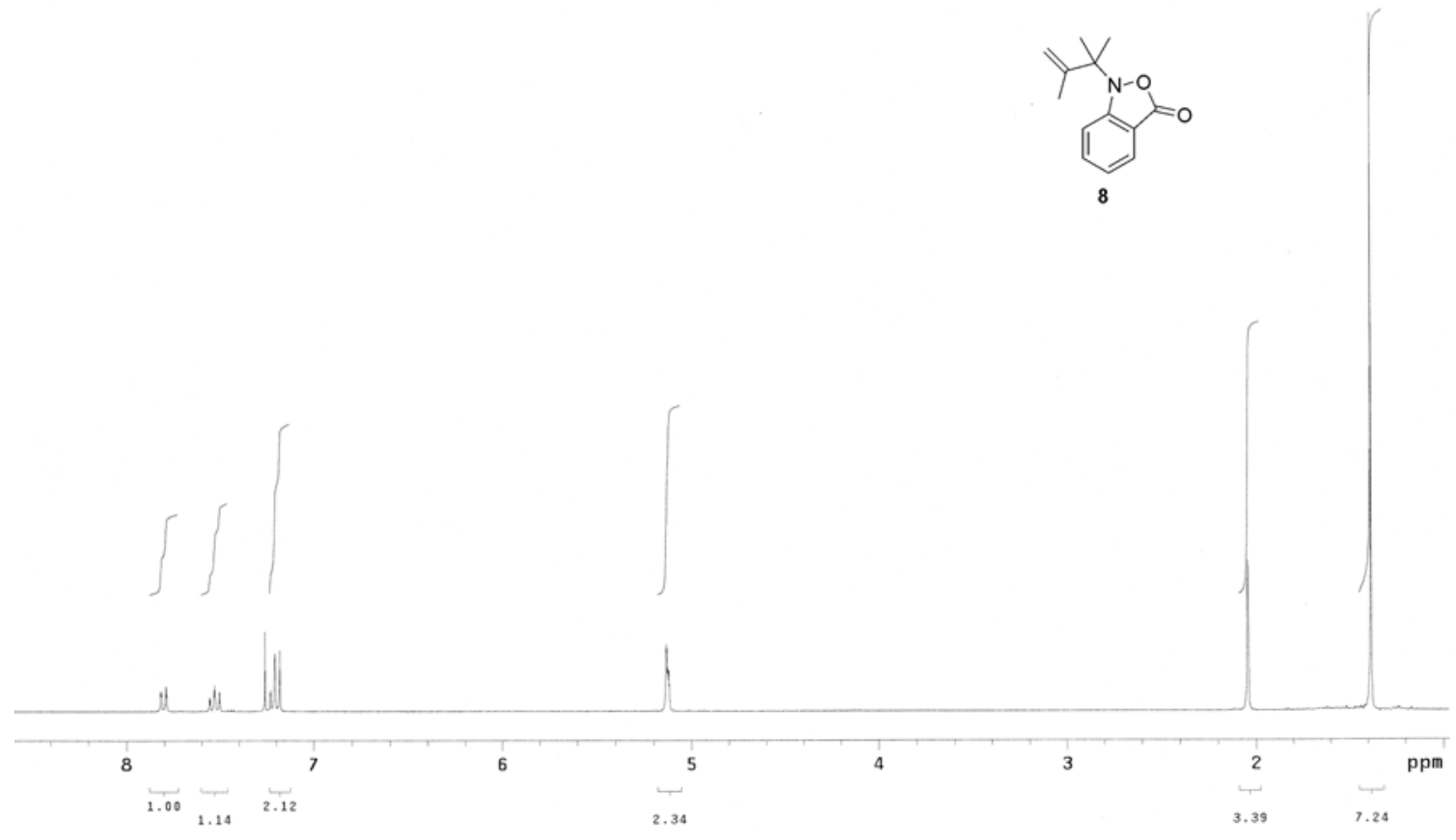

8, ${ }^{13} \mathrm{C} \mathrm{NMR}, 75 \mathrm{MHz}, \mathrm{CDCl}_{3}$

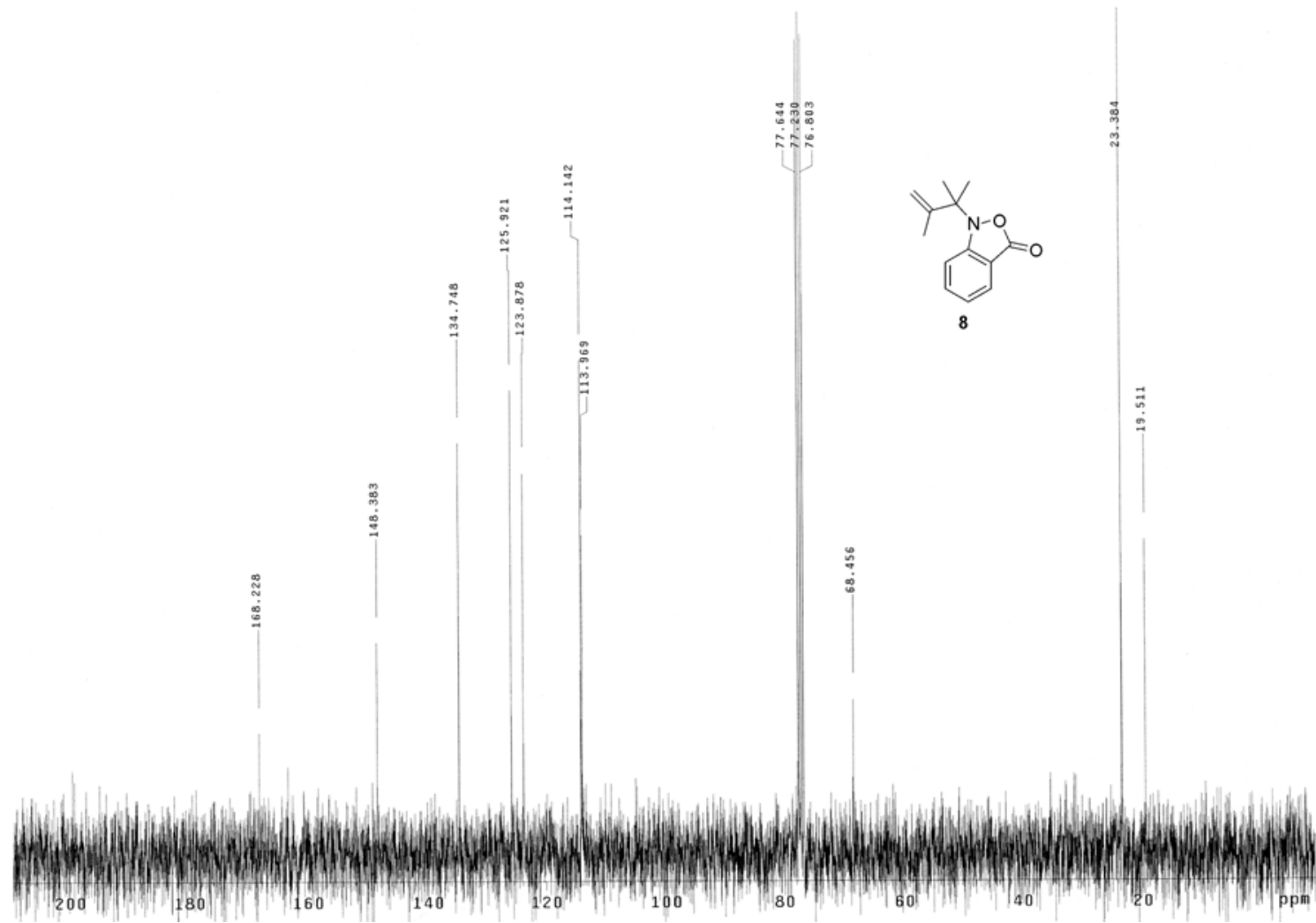


9a, ${ }^{1} \mathrm{H} \mathrm{NMR}, 300 \mathrm{MHz}, \mathrm{CDCl}_{3}$

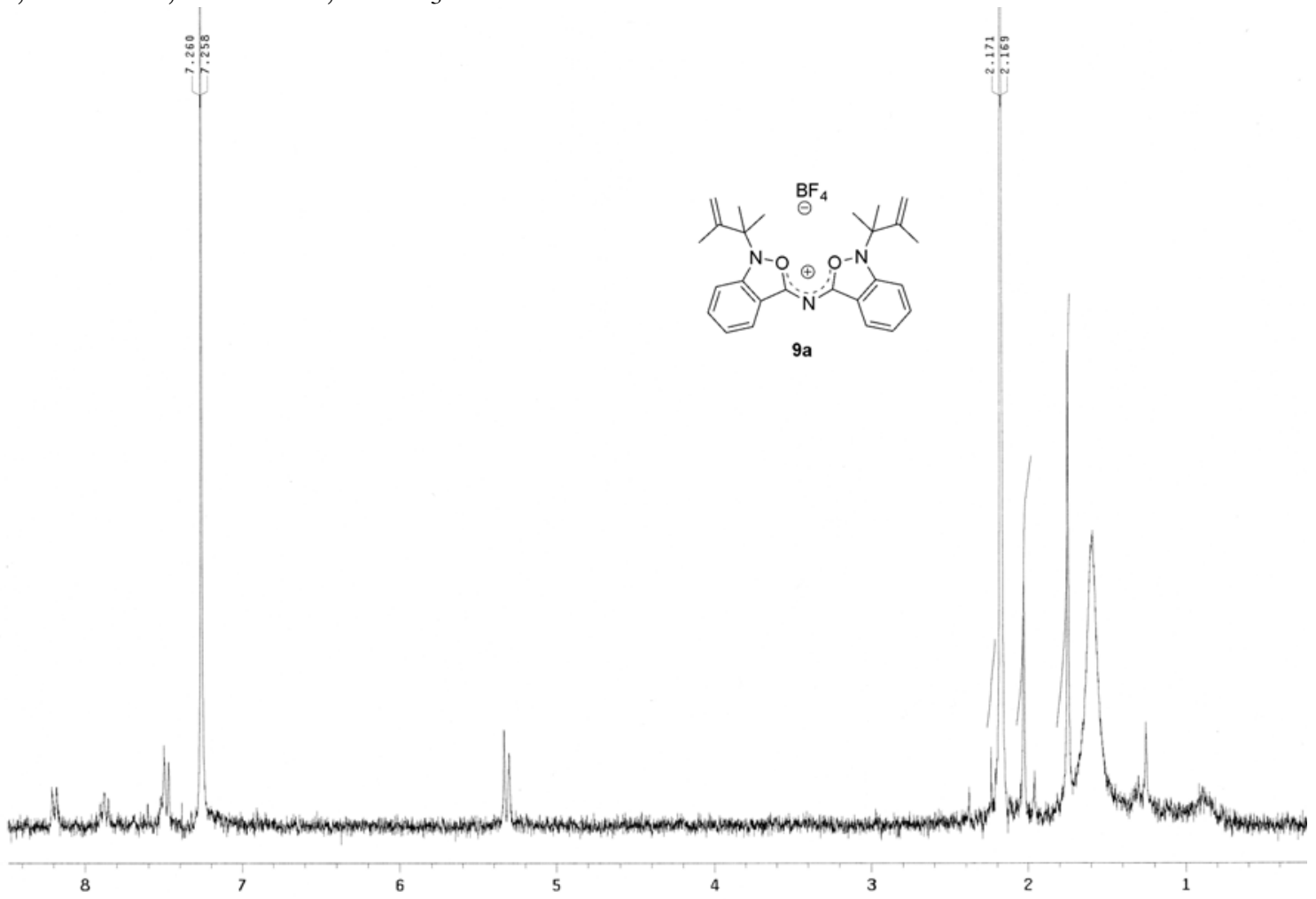

9a, ${ }^{13} \mathrm{C} \mathrm{NMR}, 75 \mathrm{MHz}, \mathrm{CDCl}_{3}$

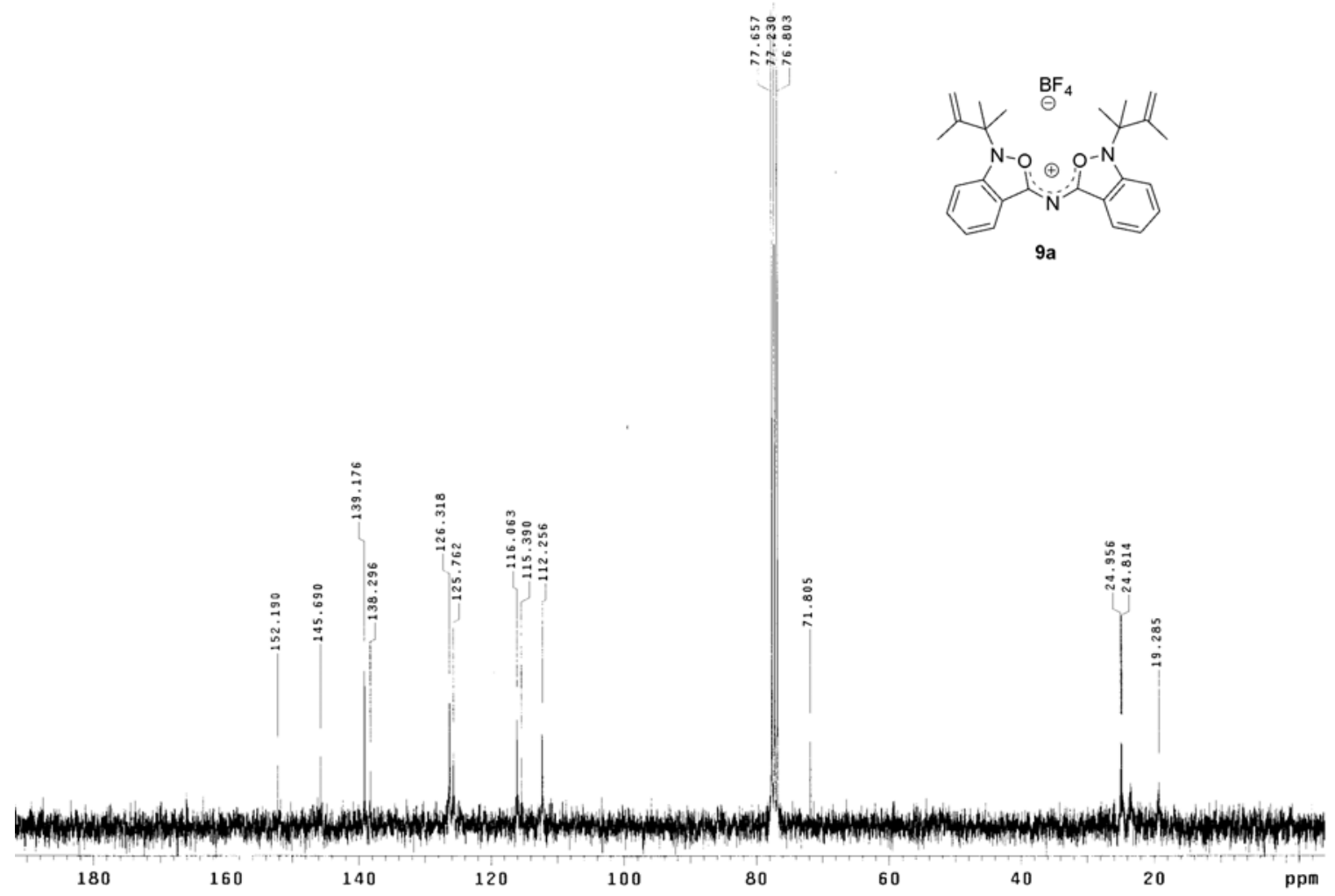


9b, ${ }^{1} \mathrm{H}$ NMR, $300 \mathrm{MHz}, \mathrm{CDCl}_{3}$

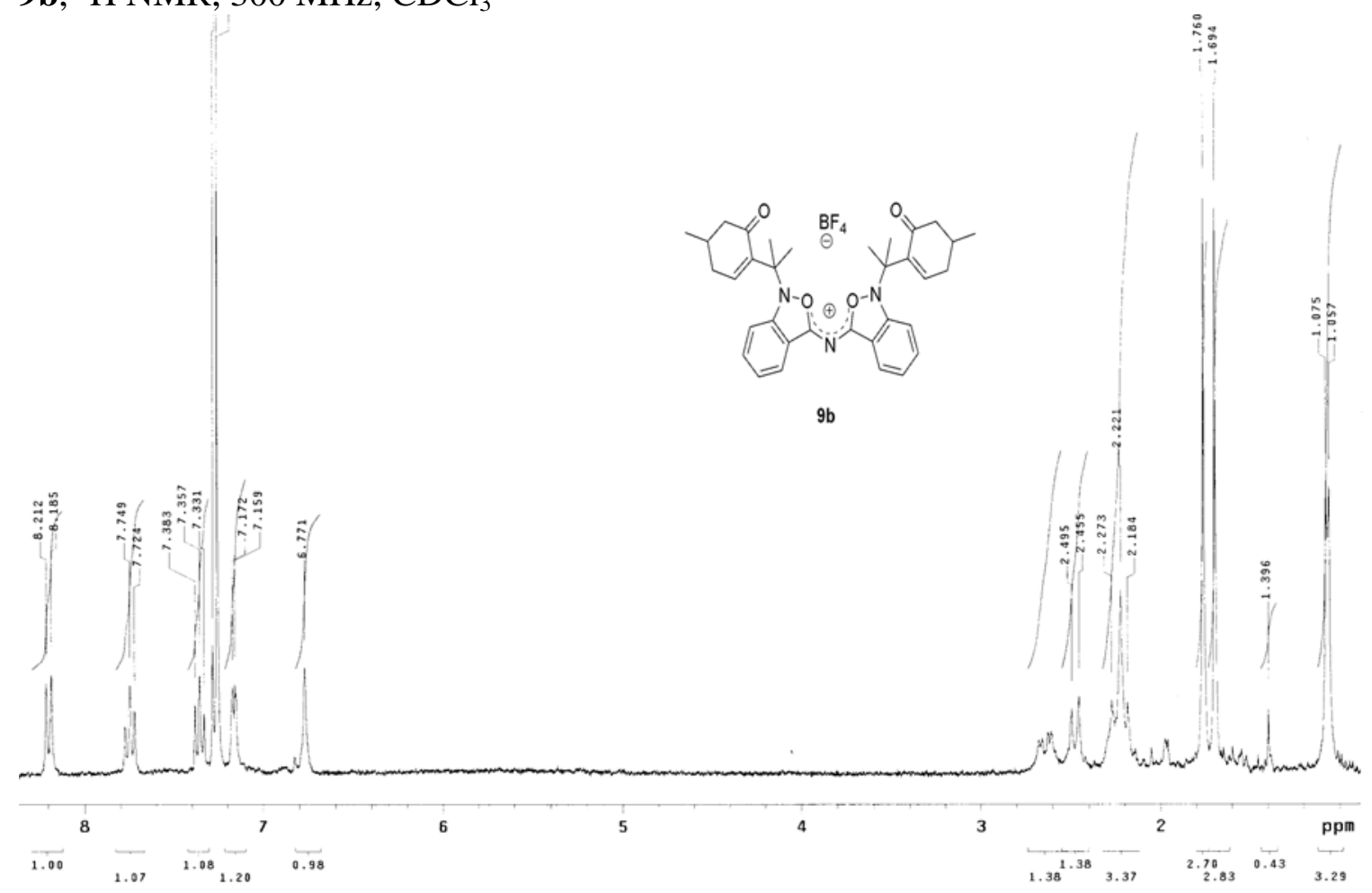

9b, ${ }^{13} \mathrm{C} \mathrm{NMR}, 75 \mathrm{MHz}, \mathrm{CDCl}_{3}$

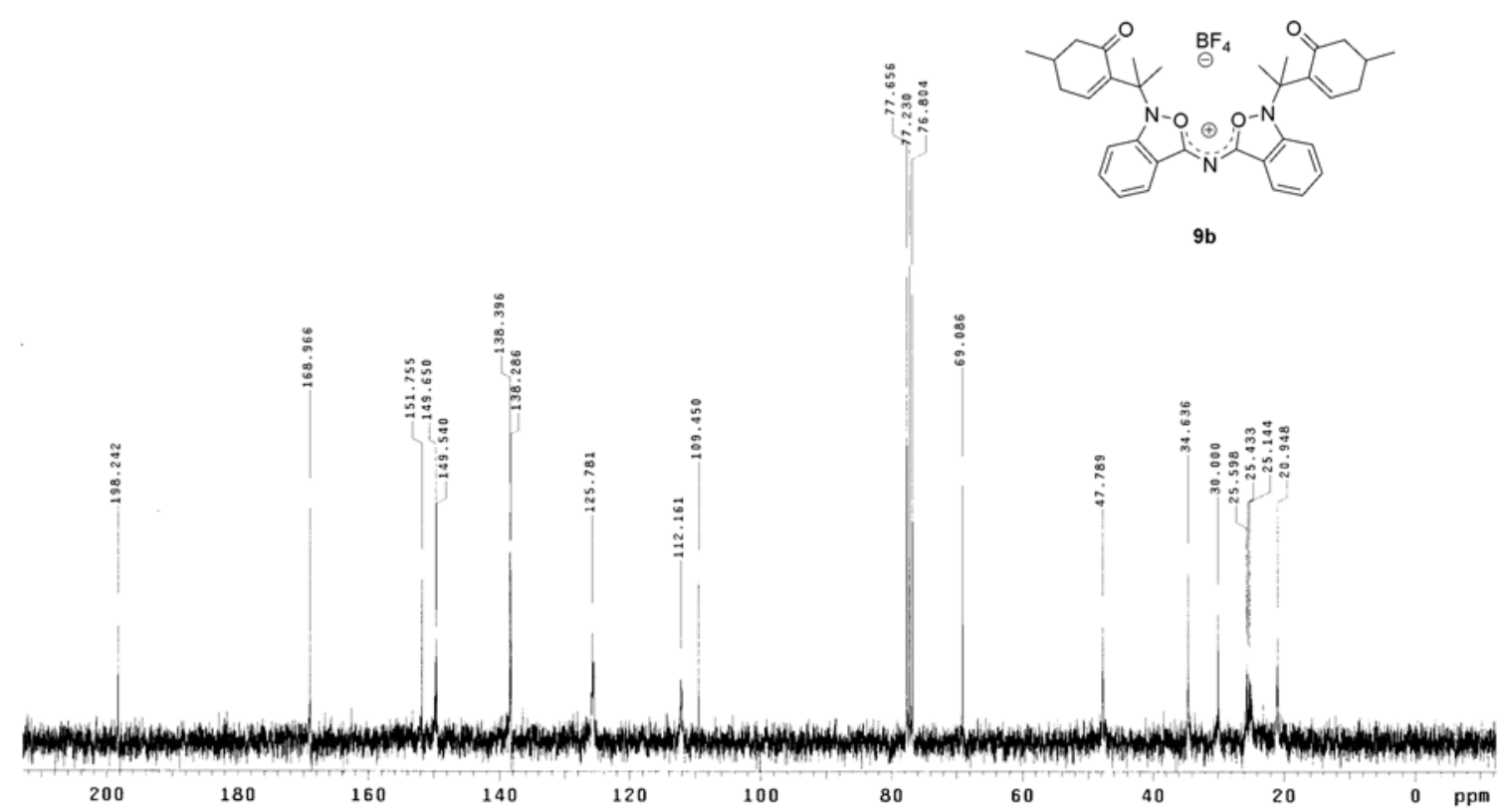


9c, ${ }^{1} \mathrm{H} \mathrm{NMR}, 300 \mathrm{MHz}, \mathrm{CDCl}_{3}$

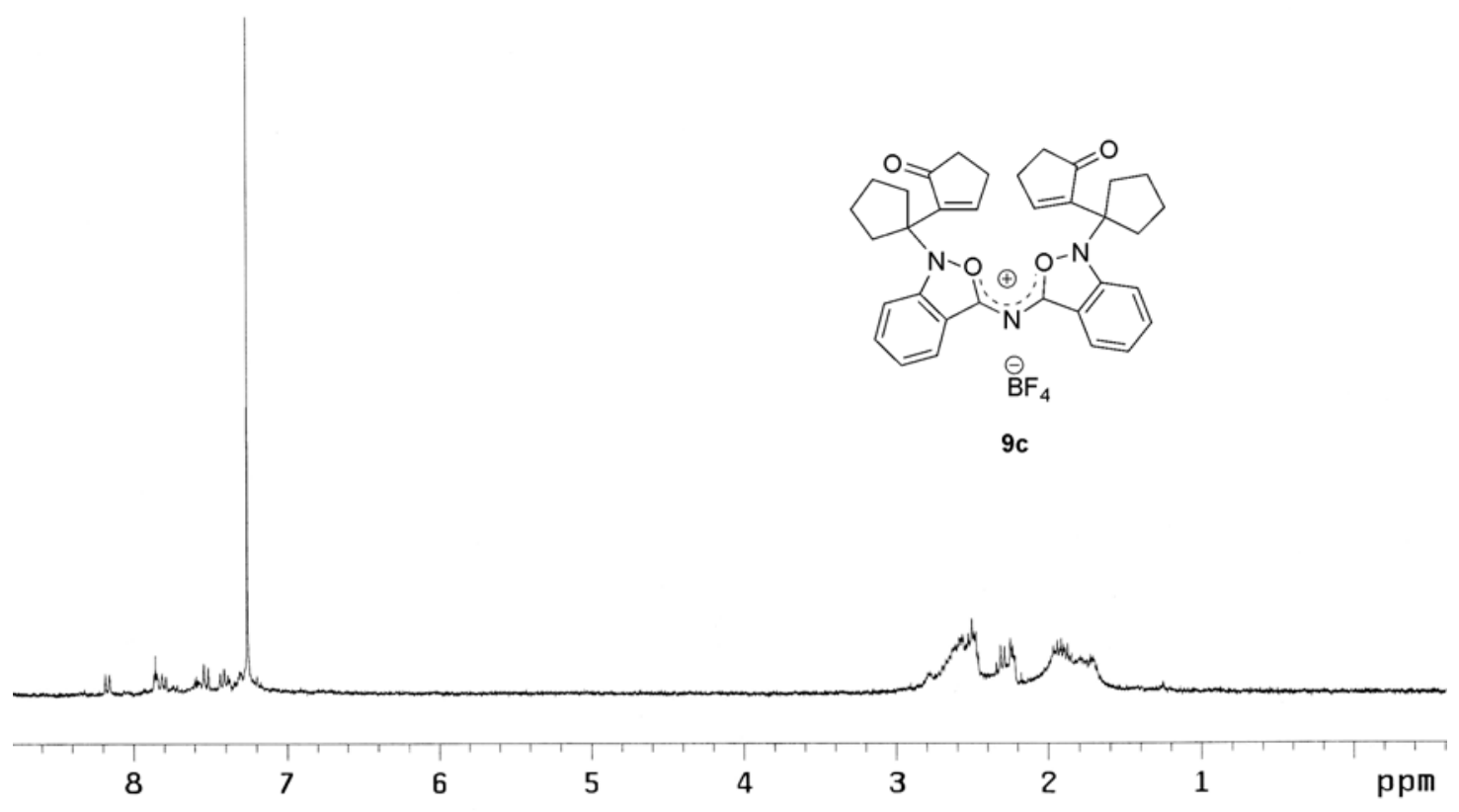

9c, ${ }^{13} \mathrm{C} \mathrm{NMR}, 75 \mathrm{MHz}, \mathrm{CDCl}_{3}$

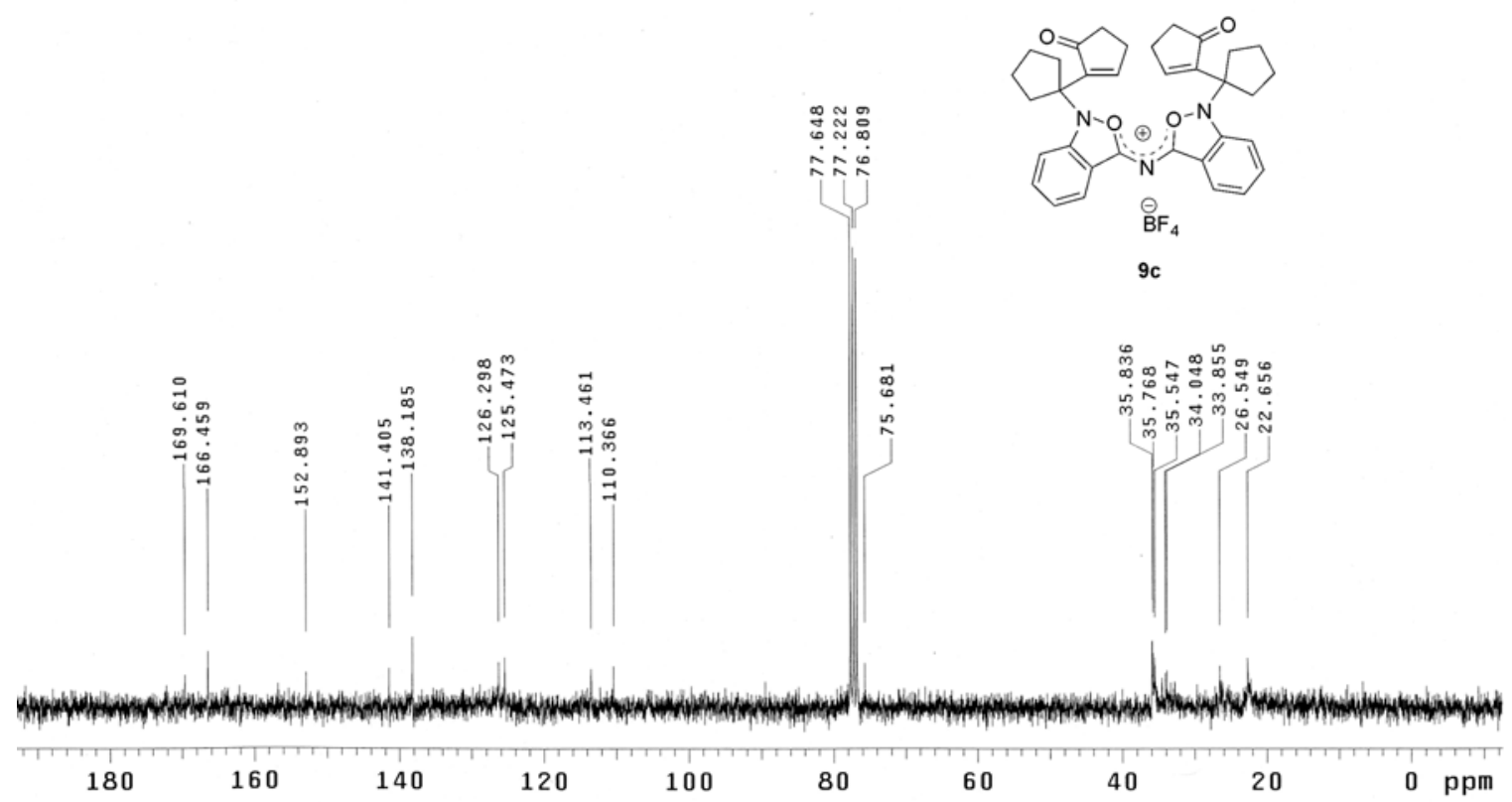

N.B. Poor solubility, the presence of residual $\mathrm{HBF}_{4}$, and minute amounts of material all contributed in the reduced quality of the NMR spectra of $9 \mathbf{c}$. 
10, ${ }^{1} \mathrm{H}$ NMR, $300 \mathrm{MHz}, \mathrm{CDCl}_{3}$

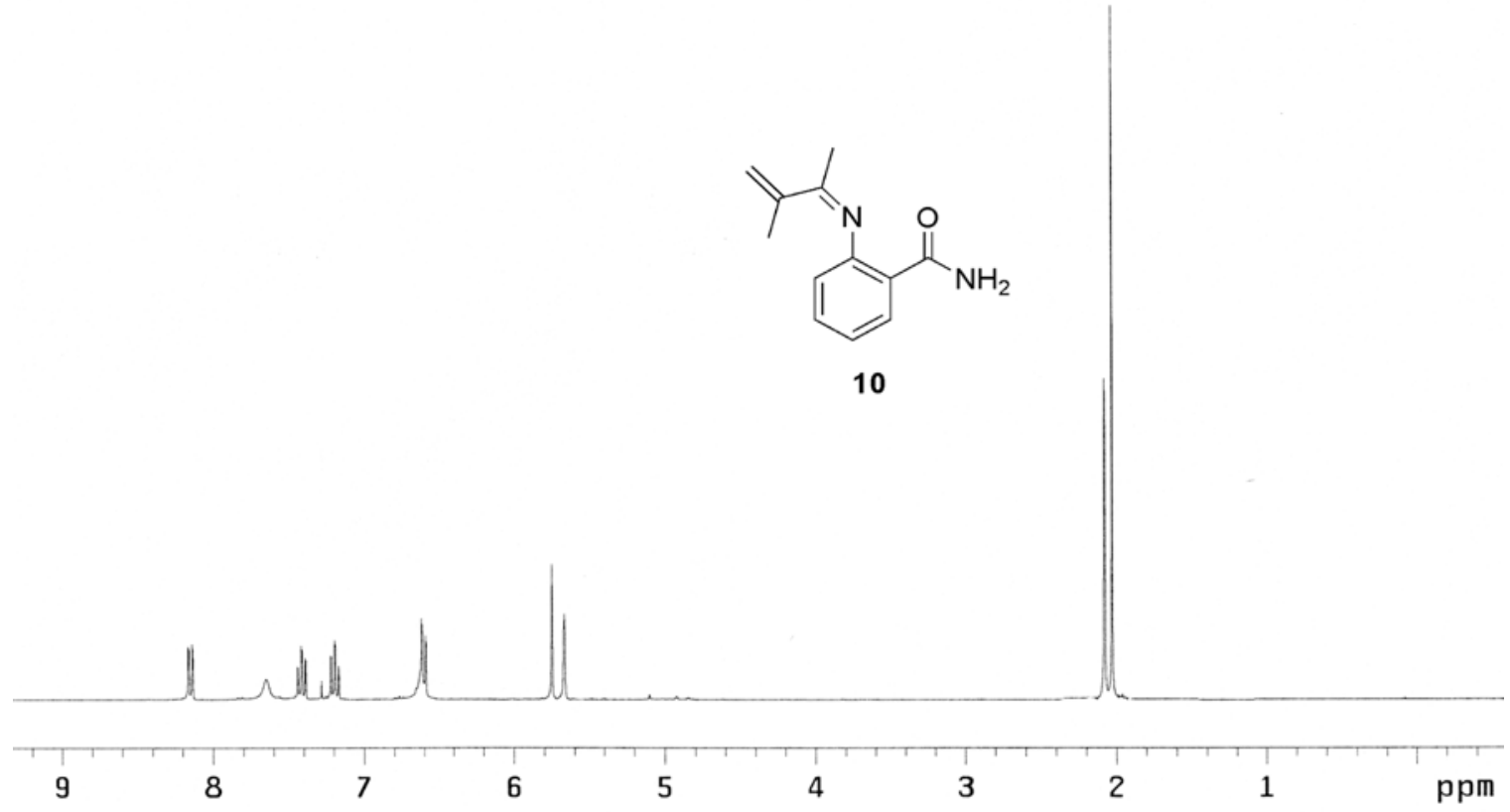

10, ${ }^{13} \mathrm{C}$ NMR, $75 \mathrm{MHz}, \mathrm{CDCl}_{3}$

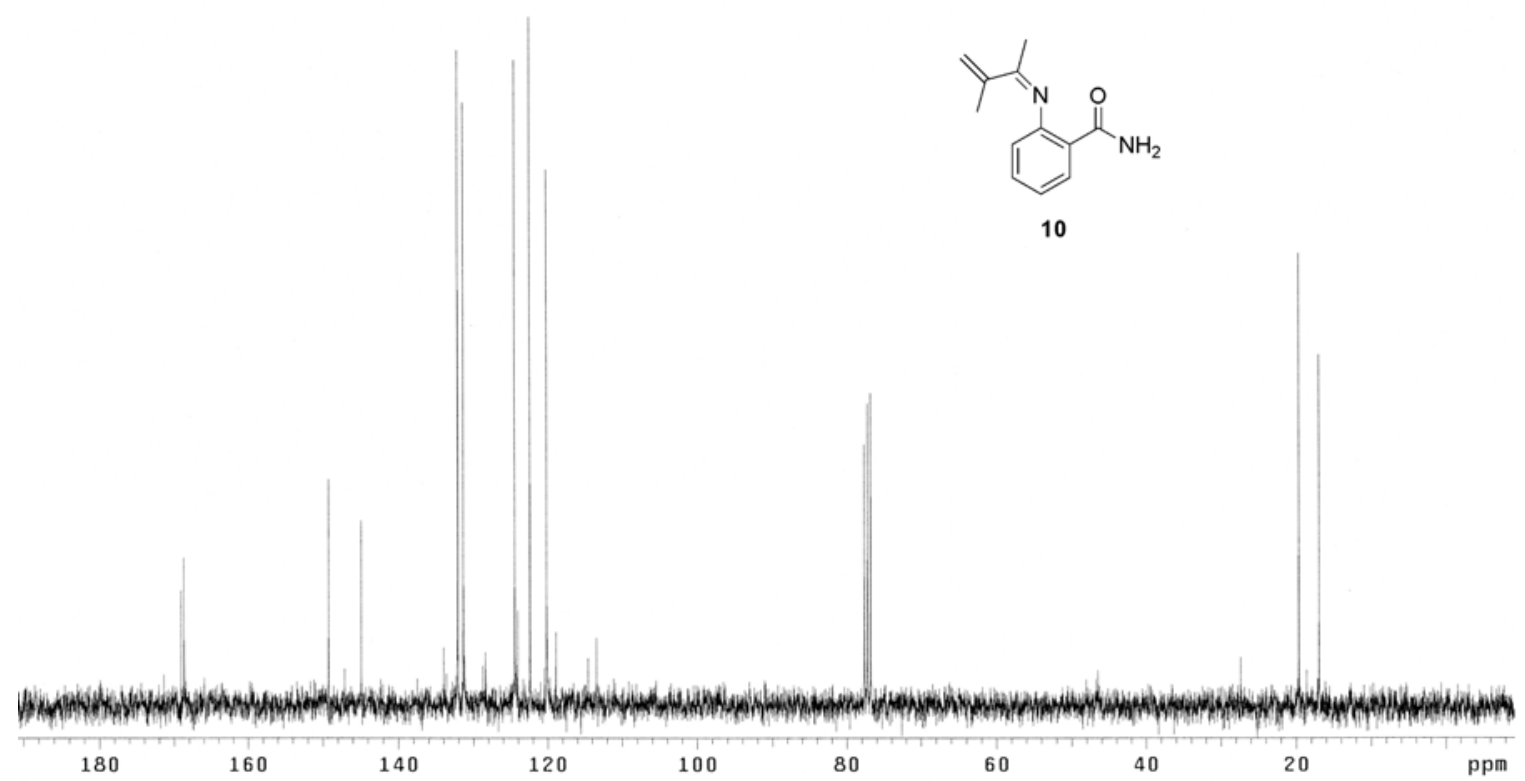




\section{Computational Studies}

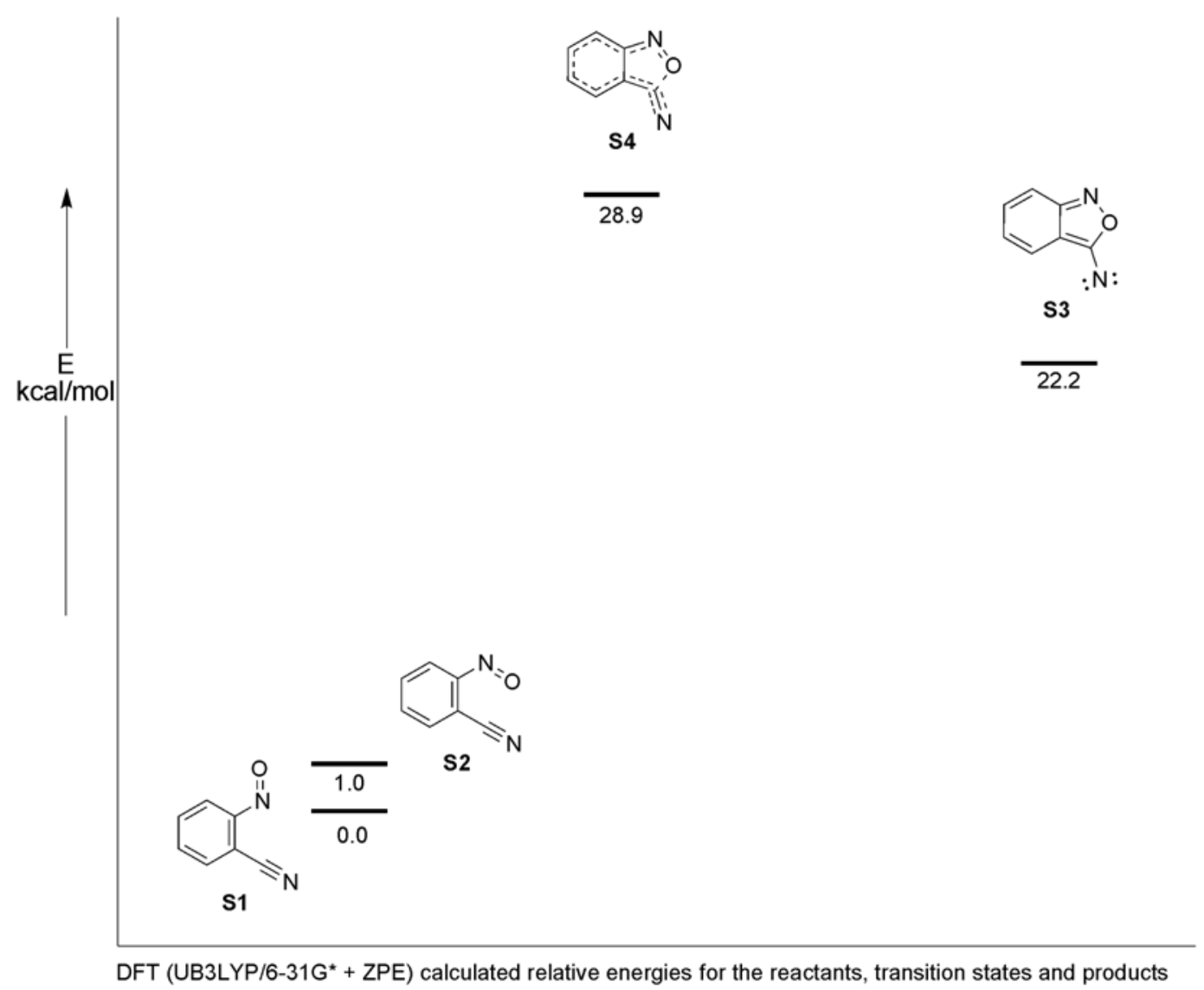

Figure S1. Energy profile for the coarctate cyclization of $o$-nitrosobenzonitrile (6).

\section{Cartesian Coordinates of Calculated Structures}

S1

Point Group: CS

UB3LYP/6-31G* $=-453.775970544 \mathrm{au}$

UB3LYP/6-31G* Zero Point Corrected Energy $=-453.680076$ au

$\mathrm{NIMAG}=0$
$\mathrm{C}$
2.33699700
0.03251800
0.00000000
$\mathrm{C}$
$\begin{array}{lll}1.98639400 & -1.32422800 & 0.00000000\end{array}$
C
$\begin{array}{llll}0.64808900 & -1.71236400 & 0.00000000\end{array}$
$\mathrm{C}$
$\begin{array}{lll}-0.36153300 & -0.73744900 & 0.00000000\end{array}$
$\mathrm{C}$
$\begin{array}{llll}0.00000000 & 0.62470500 & 0.00000000\end{array}$ 


$\begin{array}{lrrr}\mathrm{C} & 1.34473200 & 1.00561200 & 0.00000000 \\ \mathrm{~N} & -1.09308300 & 1.57144000 & 0.000000000 \\ \mathrm{C} & -1.73675300 & -1.14743700 & 0.00000000 \\ \mathrm{~N} & -2.82407100 & -1.55919400 & 0.00000000 \\ \mathrm{O} & -0.74799400 & 2.74056500 & 0.00000000 \\ \mathrm{H} & 3.38370800 & 0.32219200 & 0.00000000 \\ \mathrm{H} & 2.76223700 & -2.08412900 & 0.00000000 \\ \mathrm{H} & 0.37556300 & -2.76235400 & 0.00000000 \\ \mathrm{H} & 1.57497200 & 2.06589700 & 0.00000000\end{array}$

S2

Point Group: C1

UB3LYP/6-31G* $=-453.774374253$ au

UB3LYP/6-31G* Zero Point Corrected Energy $=-453.678513$ au $\mathrm{NIMAG}=0$

$\begin{array}{lrrr}\mathrm{C} & -2.46063000 & 0.35305500 & 0.00009400 \\ \mathrm{C} & -2.19773500 & -1.01790800 & 0.00014900 \\ \mathrm{C} & -0.88255800 & -1.48943900 & -0.00001500 \\ \mathrm{C} & 0.19707500 & -0.59561800 & -0.00040200 \\ \mathrm{C} & -0.07838900 & 0.79266800 & -0.00030200 \\ \mathrm{C} & -1.39685700 & 1.25273300 & 0.00007800 \\ \mathrm{~N} & 0.90943300 & 1.84277000 & -0.00029200 \\ \mathrm{C} & 1.52081100 & -1.14826800 & -0.00026700 \\ \mathrm{~N} & 2.51771400 & -1.74882900 & 0.00023200 \\ \mathrm{O} & 2.06731300 & 1.46286700 & 0.00042400 \\ \mathrm{H} & -3.48398800 & 0.71540700 & 0.00029200 \\ \mathrm{H} & -3.01751100 & -1.73019600 & 0.00036500 \\ \mathrm{H} & -0.6822600 & -2.55594600 & 0.00012500 \\ \mathrm{H} & -1.55511500 & 2.32687500 & 0.00023000\end{array}$

S3

Point Group: $\mathrm{C} 1$

UB3LYP/6-31G* $=-453.740602558$ au

UB3LYP/6-31G* Zero Point Corrected Energy $=-453.644662$ au $\mathrm{NIMAG}=0$ 


$\begin{array}{lrrr}\mathrm{C} & 1.52797600 & -1.26241300 & 0.00002400 \\ \mathrm{C} & 2.47744200 & -0.25516000 & 0.00000500 \\ \mathrm{C} & 2.11061700 & 1.11185700 & -0.00002900 \\ \mathrm{C} & 0.77343200 & 1.50308500 & -0.00001600 \\ \mathrm{C} & -0.19212400 & 0.49799000 & 0.00002800 \\ \mathrm{C} & 0.16935000 & -0.87338700 & 0.00000800 \\ \mathrm{C} & -1.63943300 & 0.48305000 & 0.00003700 \\ \mathrm{O} & -2.02116300 & -0.86458400 & -0.00006100 \\ \mathrm{~N} & -0.90992800 & -1.69217800 & -0.00000400 \\ \mathrm{~N} & -2.50532400 & 1.42014100 & 0.00002500 \\ \mathrm{H} & 1.79898400 & -2.31270600 & 0.00006300 \\ \mathrm{H} & 3.53166300 & -0.51628800 & 0.00001900 \\ \mathrm{H} & 2.88922800 & 1.86850100 & -0.00007300 \\ \mathrm{H} & 0.49263000 & 2.55129000 & -0.00001600\end{array}$

\section{S4}

Point Group: $\mathrm{C} 1$

UB3LYP/6-31G* $=-453.728072975$ au UB3LYP/6-31G* Zero Point Corrected Energy $=-453.633958$ au $\mathrm{NIMAG}=1$

$\begin{array}{lrrr}\mathrm{C} & -2.48416900 & -0.27286500 & -0.00001000 \\ \mathrm{C} & -2.13965600 & 1.09397600 & 0.00001500 \\ \mathrm{C} & -0.80465600 & 1.49643700 & 0.00002800 \\ \mathrm{C} & 0.18754100 & 0.51496200 & 0.00002000 \\ \mathrm{C} & -0.16380200 & -0.86248800 & 0.00000300 \\ \mathrm{C} & -1.51237300 & -1.26305000 & -0.00001200 \\ \mathrm{~N} & 0.91922800 & -1.68848200 & 0.00000700 \\ \mathrm{C} & 1.61530200 & 0.67990900 & -0.00000900 \\ \mathrm{~N} & 2.55228000 & 1.45160100 & -0.00007100 \\ \mathrm{O} & 2.03380000 & -1.02271800 & 0.00002600 \\ \mathrm{H} & -3.53269000 & -0.55587200 & -0.00002000 \\ \mathrm{H} & -2.92425500 & 1.84410600 & 0.00002300 \\ \mathrm{H} & -0.53729800 & 2.54805800 & 0.00005000 \\ \mathrm{H} & -1.76583000 & -2.31766600 & -0.00002500\end{array}$


Cartesian Coordinates of Calculated Structures in Figure 2

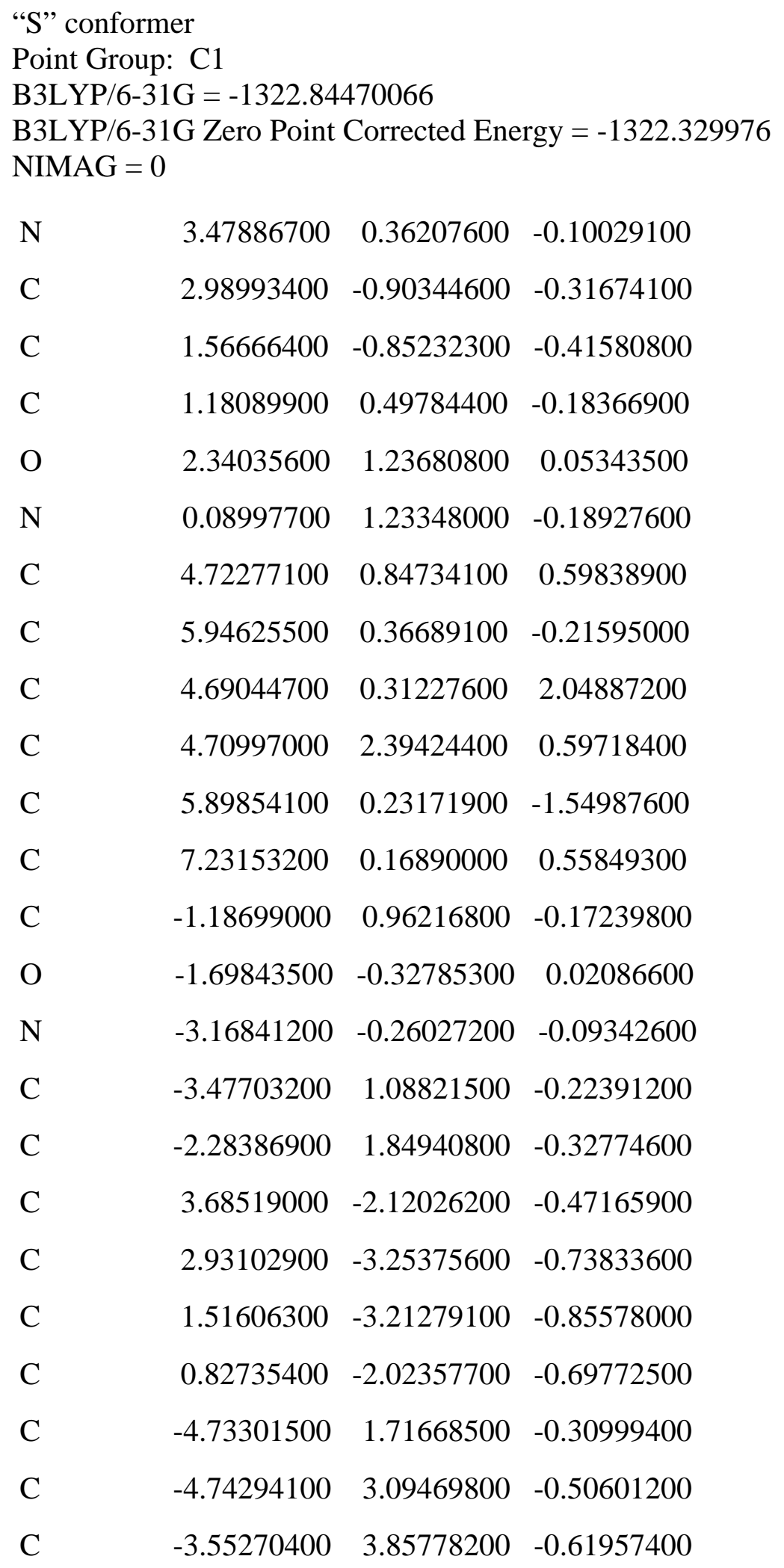




\begin{tabular}{|c|c|c|c|}
\hline $\mathrm{C}$ & -2.31474300 & 3.24187500 & -0.53168700 \\
\hline $\mathrm{C}$ & -3.86157100 & -1.31667500 & 0.74421000 \\
\hline $\mathrm{C}$ & -3.83797300 & -0.87797200 & 2.22080900 \\
\hline $\mathrm{C}$ & -3.08290900 & -2.64243900 & 0.57545300 \\
\hline $\mathrm{C}$ & -5.27574400 & -1.47426000 & 0.14461000 \\
\hline $\mathrm{C}$ & -5.36465900 & -1.82242100 & -1.32760300 \\
\hline $\mathrm{C}$ & -6.37573800 & -1.38124200 & 0.90870800 \\
\hline $\mathrm{H}$ & 4.71958900 & -0.78133600 & 2.08139100 \\
\hline $\mathrm{H}$ & 5.54153400 & 0.69494500 & 2.61697500 \\
\hline $\mathrm{H}$ & 3.77647000 & 0.65407600 & 2.54437600 \\
\hline $\mathrm{H}$ & 4.59240800 & 2.77853000 & -0.41835600 \\
\hline $\mathrm{H}$ & 3.90940800 & 2.78937800 & 1.22666700 \\
\hline $\mathrm{H}$ & 5.66703400 & 2.74808600 & 0.99040600 \\
\hline $\mathrm{H}$ & 6.78429000 & -0.03910800 & -2.11510900 \\
\hline $\mathrm{H}$ & 4.99153300 & 0.39280800 & -2.12232900 \\
\hline $\mathrm{H}$ & 8.05495900 & -0.05199900 & -0.12502400 \\
\hline $\mathrm{H}$ & 7.50735700 & 1.06544400 & 1.12880200 \\
\hline $\mathrm{H}$ & 7.16026700 & -0.65683200 & 1.27687400 \\
\hline $\mathrm{H}$ & 4.76263400 & -2.15957700 & -0.40814600 \\
\hline $\mathrm{H}$ & 3.44054400 & -4.20284500 & -0.86498700 \\
\hline $\mathrm{H}$ & 0.97642300 & -4.12748900 & -1.07033600 \\
\hline $\mathrm{H}$ & -0.25028200 & -1.98172700 & -0.77635200 \\
\hline $\mathrm{H}$ & -5.65087600 & 1.15340600 & -0.22558200 \\
\hline $\mathrm{H}$ & -5.69896300 & 3.60254000 & -0.57469100 \\
\hline $\mathrm{H}$ & -3.61987900 & 4.92779600 & -0.77499500 \\
\hline $\mathrm{H}$ & -1.38798300 & 3.79732900 & -0.61121900 \\
\hline $\mathrm{H}$ & -2.80312600 & -0.72591500 & 2.54216700 \\
\hline $\mathrm{H}$ & -4.39336800 & 0.05106900 & 2.37776700 \\
\hline $\mathrm{H}$ & -4.27413900 & -1.65444100 & 2.85508200 \\
\hline
\end{tabular}




$\begin{array}{lrrr}\mathrm{H} & -2.93391400 & -2.88830400 & -0.47839800 \\ \mathrm{H} & -3.66192700 & -3.44477700 & 1.04129200 \\ \mathrm{H} & -2.10866700 & -2.58868100 & 1.06678600 \\ \mathrm{H} & -4.80762900 & -1.11334500 & -1.95012300 \\ \mathrm{H} & -6.40650600 & -1.82341900 & -1.65812800 \\ \mathrm{H} & -4.95443900 & -2.81872200 & -1.53368700 \\ \mathrm{H} & -7.35922500 & -1.55930500 & 0.48534000 \\ \mathrm{H} & -6.35248500 & -1.14950500 & 1.96653200\end{array}$

"W" conformer

Point Group: C1

B3LYP/6-31G = -1322.83501651

B3LYP/6-31G Zero Point Corrected Energy = -1322.320237

$\mathrm{NIMAG}=0$
$\mathrm{N} \quad-3.64701200-0.20744500 \quad-0.14600600$
C $\quad-3.28551800 \quad 1.04341400 \quad-0.59300400$
C $\quad-1.86383400 \quad 1.11979600 \quad-0.67941100$
C $\quad-1.34268100-0.12301700 \quad-0.21335300$
$\begin{array}{llll}\text { O } & -2.41686500 & -0.92882600 & 0.14348000\end{array}$
$\mathrm{N} \quad \quad-0.16863500 \quad-0.68676800 \quad-0.14890800$
C $\quad-4.80346000 \quad-0.78292900 \quad 0.64031700$
$\begin{array}{llll}\text { C } & -4.84818900 & -2.29211900 & 0.33094900\end{array}$
C $\quad-6.10561000 \quad-0.11144600 \quad 0.13777800$
C $\quad-4.58607000 \quad-0.44591500 \quad 2.12938000$
C $\quad-4.89866500 \quad-3.20794200 \quad 1.30809400$
C $\quad-4.88994200 \quad-2.68746000 \quad-1.12960500$
$\begin{array}{llll}\text { C } & 1.06033100 & -0.28796400 & 0.02494500\end{array}$
O $\quad 2.04183100-1.12415100 \quad-0.49671200$
$\mathrm{N} \quad 3.34007900 \quad-0.63565300 \quad-0.05166500$
$\begin{array}{llll}\text { C } & 3.11288800 & 0.54246100 & 0.62318800\end{array}$
$\begin{array}{llll}\text { C } & 1.71135600 & 0.76548400 & 0.73373800\end{array}$ 


\begin{tabular}{|c|c|c|c|}
\hline $\mathrm{C}$ & -4.07638600 & 2.13822100 & -1.00635500 \\
\hline $\mathrm{C}$ & -3.41995600 & 3.26234400 & -1.48894500 \\
\hline $\mathrm{C}$ & -2.00662500 & 3.33714300 & -1.58741500 \\
\hline $\mathrm{C}$ & -1.22317900 & 2.26728800 & -1.19126100 \\
\hline $\mathrm{C}$ & 4.03107800 & 1.43590600 & 1.21339700 \\
\hline $\mathrm{C}$ & 3.50941100 & 2.51529200 & 1.91413500 \\
\hline $\mathrm{C}$ & 2.11289100 & 2.73017500 & 2.05171400 \\
\hline $\mathrm{C}$ & 1.20789900 & 1.85978200 & 1.46773000 \\
\hline $\mathrm{C}$ & 4.48050200 & -1.08925400 & -0.93333300 \\
\hline $\mathrm{C}$ & 4.52567600 & -0.18023600 & -2.17759400 \\
\hline $\mathrm{C}$ & 4.19844700 & -2.55071300 & -1.35836500 \\
\hline $\mathrm{C}$ & 5.75072600 & -1.04171200 & -0.05782600 \\
\hline $\mathrm{C}$ & 5.73773100 & -1.86365000 & 1.21517900 \\
\hline $\mathrm{C}$ & 6.84582100 & -0.36835100 & -0.44519500 \\
\hline $\mathrm{H}$ & -6.19510100 & -0.14146200 & -0.95060500 \\
\hline $\mathrm{H}$ & -6.94730600 & -0.66611600 & 0.56115700 \\
\hline $\mathrm{H}$ & -6.18430800 & 0.92266800 & 0.48390800 \\
\hline $\mathrm{H}$ & -3.68453000 & -0.92559200 & 2.51751800 \\
\hline $\mathrm{H}$ & -4.49546300 & 0.63798000 & 2.25536900 \\
\hline $\mathrm{H}$ & -5.44243600 & -0.77994300 & 2.72132300 \\
\hline $\mathrm{H}$ & -4.97885500 & -4.26379900 & 1.07173500 \\
\hline $\mathrm{H}$ & -4.86572200 & -2.96288800 & 2.36250800 \\
\hline $\mathrm{H}$ & -4.91622800 & -3.77550200 & -1.22853700 \\
\hline $\mathrm{H}$ & -5.77431700 & -2.28809400 & -1.64175100 \\
\hline $\mathrm{H}$ & -4.00986000 & -2.32111200 & -1.66998200 \\
\hline $\mathrm{H}$ & -5.15429300 & 2.10773500 & -0.97232500 \\
\hline $\mathrm{H}$ & -4.01316500 & 4.11054100 & -1.81309100 \\
\hline $\mathrm{H}$ & -1.54666800 & 4.23368300 & -1.98497800 \\
\hline $\mathrm{H}$ & 5.09659900 & 1.27925700 & 1.12614200 \\
\hline
\end{tabular}




$\begin{array}{lrrr}\mathrm{H} & 4.19497000 & 3.21548700 & 2.37905100 \\ \mathrm{H} & 1.75964100 & 3.58034300 & 2.62278400 \\ \mathrm{H} & 3.56693100 & -0.23170800 & -2.70219300 \\ \mathrm{H} & 4.72688000 & 0.86219300 & -1.91289700 \\ \mathrm{H} & 5.30275600 & -0.51940200 & -2.86783000 \\ \mathrm{H} & 3.34707300 & -2.60265800 & -2.03987100 \\ \mathrm{H} & 3.98901200 & -3.18844700 & -0.49756600 \\ \mathrm{H} & 5.08363900 & -2.93420800 & -1.87345400 \\ \mathrm{H} & 5.70433600 & -2.93844500 & 0.99976300 \\ \mathrm{H} & 4.86722400 & -1.63803400 & 1.84132100 \\ \mathrm{H} & 6.64006900 & -1.67508800 & 1.80255900 \\ \mathrm{H} & 7.75347200 & -0.40053900 & 0.14915500 \\ \mathrm{H} & 6.90025300 & 0.21152300 & -1.35850500 \\ \mathrm{H} & 0.14017800 & 2.01188400 & 1.57350600 \\ \mathrm{H} & -0.14339300 & 2.30505000 & -1.27559800\end{array}$

"U" conformer

Point Group: C1

B3LYP/6-31G $=-1322.84699945$

B3LYP/6-31G Zero Point Corrected Energy $=-1322.332865$

$\mathrm{NIMAG}=0$
$\begin{array}{llll}\text { C } & -4.82046700 & 1.23398900 & -0.07393800\end{array}$
$\begin{array}{llll}\text { C } & -5.14121300 & 2.58465600 & 0.00449700\end{array}$
$\begin{array}{llll}\text { C } & -4.15286900 & 3.60243100 & 0.06712700\end{array}$
$\begin{array}{llll}\text { C } & -2.80777500 & 3.27508600 & 0.05885700\end{array}$
$\begin{array}{llll}\text { C } & -2.46047500 & 1.91159300 & -0.00975500\end{array}$
$\begin{array}{llll}\text { C } & -3.45295200 & 0.89714200 & -0.08519900\end{array}$
$\begin{array}{llll}\text { C } & -1.19608400 & 1.27312900 & -0.00610400\end{array}$
$\begin{array}{llll}\mathrm{O} & -1.40214200 & -0.10561900 & -0.06641200\end{array}$
$\mathrm{N} \quad-2.84252300 \quad-0.33768600 \quad-0.20690100$
$\begin{array}{llll}\text { C } & -3.28139300 & -1.69087900 & 0.28591400\end{array}$ 


\begin{tabular}{|c|c|c|c|}
\hline$C$ & -3.53410100 & -1.59569600 & 1.80806200 \\
\hline $\mathrm{C}$ & -2.15358200 & -2.70644400 & -0.01014800 \\
\hline $\mathrm{C}$ & -4.53164600 & -2.10221700 & -0.52669900 \\
\hline $\mathrm{C}$ & -4.70720100 & -1.71393100 & -1.79895800 \\
\hline $\mathrm{C}$ & -5.49057700 & -3.05617800 & 0.15291600 \\
\hline $\mathrm{N}$ & 0.00000000 & 1.82485000 & 0.01543100 \\
\hline $\mathrm{C}$ & 1.19608600 & 1.27313100 & -0.00607500 \\
\hline $\mathrm{C}$ & 2.46047800 & 1.91158600 & -0.00957200 \\
\hline $\mathrm{C}$ & 3.45295600 & 0.89713400 & -0.08504600 \\
\hline $\mathrm{N}$ & 2.84252300 & -0.33767000 & -0.20691400 \\
\hline $\mathrm{O}$ & 1.40214700 & -0.10561600 & -0.06651000 \\
\hline $\mathrm{C}$ & 2.80778300 & 3.27507100 & 0.05919100 \\
\hline $\mathrm{C}$ & 4.15287800 & 3.60240600 & 0.06759200 \\
\hline $\mathrm{C}$ & 5.14122100 & 2.58462800 & 0.00494100 \\
\hline $\mathrm{C}$ & 4.82047300 & 1.23397200 & -0.07364700 \\
\hline $\mathrm{C}$ & 3.28135700 & -1.69095400 & 0.28567000 \\
\hline $\mathrm{C}$ & 2.15358700 & -2.70647400 & -0.01070700 \\
\hline $\mathrm{C}$ & 3.53390500 & -1.59606900 & 1.80786300 \\
\hline $\mathrm{C}$ & 4.53169200 & -2.10211000 & -0.52689800 \\
\hline $\mathrm{C}$ & 5.49062300 & -3.05613100 & 0.15263800 \\
\hline $\mathrm{C}$ & 4.70731900 & -1.71363900 & -1.79909200 \\
\hline $\mathrm{H}$ & -5.58667900 & 0.47601500 & -0.14343200 \\
\hline $\mathrm{H}$ & -6.18742300 & 2.87087100 & 0.01394500 \\
\hline $\mathrm{H}$ & -4.46191600 & 4.63936700 & 0.12078100 \\
\hline $\mathrm{H}$ & -2.03189000 & 4.02954000 & 0.10624800 \\
\hline $\mathrm{H}$ & -4.34988300 & -0.90507700 & 2.04268000 \\
\hline $\mathrm{H}$ & -2.62585700 & -1.24643800 & 2.30930000 \\
\hline $\mathrm{H}$ & -3.78697000 & -2.57642900 & 2.21821600 \\
\hline $\mathrm{H}$ & -1.86865200 & -2.66819000 & -1.06387000 \\
\hline
\end{tabular}




\begin{tabular}{|c|c|c|c|}
\hline $\mathrm{H}$ & -1.27355100 & -2.51564800 & 0.60844600 \\
\hline $\mathrm{H}$ & -2.52279100 & -3.71165700 & 0.21137100 \\
\hline $\mathrm{H}$ & -5.55574700 & -2.06575600 & -2.37667200 \\
\hline $\mathrm{H}$ & -4.01974400 & -1.04631500 & -2.30648600 \\
\hline $\mathrm{H}$ & -5.98447000 & -2.60094000 & 1.01988000 \\
\hline $\mathrm{H}$ & -4.98313200 & -3.96071800 & 0.51298500 \\
\hline $\mathrm{H}$ & -6.26818100 & -3.37228600 & -0.54672800 \\
\hline $\mathrm{H}$ & 2.03190000 & 4.02952600 & 0.10659600 \\
\hline $\mathrm{H}$ & 4.46192800 & 4.63933400 & 0.12136600 \\
\hline $\mathrm{H}$ & 6.18743200 & 2.87083600 & 0.01449300 \\
\hline $\mathrm{H}$ & 5.58668800 & 0.47600200 & -0.14315900 \\
\hline $\mathrm{H}$ & 1.27347900 & -2.51579300 & 0.60781200 \\
\hline $\mathrm{H}$ & 1.86878400 & -2.66803600 & -1.06445600 \\
\hline $\mathrm{H}$ & 2.52277600 & -3.71172400 & 0.21067900 \\
\hline $\mathrm{H}$ & 4.34966400 & -0.90549600 & 2.04270200 \\
\hline $\mathrm{H}$ & 2.62561000 & -1.24690300 & 2.30907000 \\
\hline $\mathrm{H}$ & 3.78672500 & -2.57688300 & 2.21785400 \\
\hline $\mathrm{H}$ & 5.98427400 & -2.60107400 & 1.01983400 \\
\hline $\mathrm{H}$ & 4.98323300 & -3.96084900 & 0.51234100 \\
\hline $\mathrm{H}$ & 6.26841400 & -3.37192500 & -0.54694000 \\
\hline $\mathrm{H}$ & 5.55592700 & -2.06533900 & -2.37679100 \\
\hline & 4.01986900 & -1.04598400 & -2.30657800 \\
\hline
\end{tabular}

